Research Article

\title{
Prediction of Lift Coefficient for Tandem Wing Configuration or Multiple-Lifting-Surface System Using Prandtl's Lifting-Line Theory
}

\author{
Hao Cheng $(\mathbb{D}$ and Hua Wang $\mathbb{D}$ \\ School of Astronautics, Beihang University, Beijing 100191, China \\ Correspondence should be addressed to Hao Cheng; chenghao@buaa.edu.cn
}

Received 3 December 2017; Accepted 3 June 2018; Published 8 July 2018

Academic Editor: William W. Liou

Copyright (c) 2018 Hao Cheng and Hua Wang. This is an open access article distributed under the Creative Commons Attribution License, which permits unrestricted use, distribution, and reproduction in any medium, provided the original work is properly cited.

\begin{abstract}
In tandem airfoil configuration or multiple-lifting-surface layouts, due to the flow interaction among their lifting surfaces, the aerodynamic characteristics can be affected by each other. In accordance with Prandtl's classical lifting-line theory, a method to calculate the section lift coefficient for the tandem wing configuration or multiple-lifting-surface system is presented. In that method, the form of Fourier sine series is used to express the variation of the section circulation which changes continuously along the wingspan. The accuracy of the numerical solutions obtained by the method has been validated by the data obtained from computational fluid dynamics and tunnel experiment. By varying the design parameters, such as the gap, the stagger, the incidence angle, the wingspan, the taper ratio as well as the aspect ratio, a series of tandem wing configurations are tested to analyze the lift coefficient and the induced drag of each lifting surface. From the results, it can be seen that the bigger negative gap and stagger can produce better lift characteristic for tandem wing configuration. Besides, it will also be beneficial for the lift characteristic when the incidence angle and the wingspan of fore wing are appropriately declined or if the incidence angle and the wingspan of hind wing are appropriately increased.
\end{abstract}

\section{Introduction}

Compared to the traditional layout, the induced drag for tandem airfoil configuration is smaller and the wingspan can be reduced with the same stiffness, for the reason that the two wings in tandem airfoil configuration are all generating positive lift. Researches on tandem airfoil configuration with its unique aerodynamic advantage were conducted for decades. Bottomley $[1,2]$ summarized the history of aircrafts with tandem airfoil layouts and pointed out the advantages and disadvantages of that kind of design. To study the aerodynamic characteristics for tandem airfoil configuration, three different methods, including tunnel experiment, computational fluid dynamics (CFD) method, and theoretical calculation, were extensively used.

Through experiments, Feistal et al. [3] conducted experiments on a series of multiwing configuration with finite span wings at Reynolds number of $1.4 e 6$, and the results indicated that the interaction between the two wings was favorable to the fore wing (the maximum lift coefficient increased), while the maximum lift coefficient of the hind wing did not decrease. Scharpf and Mueller [4] conducted wind tunnel experiments on different tandem wing configurations, to investigate the influence on the tandem wing aerodynamic characteristics by the horizontal distance, vertical distance, and the decalage between the two wings. Results obtained from tunnel experiments agree well with the real situation but with enormous financial costs.

Another high-fidelity method, CFD, has also been widely used to simulate the aerodynamic characteristics for different layouts. Alley et al. [5] presented a method, which used a correlation obtained from grid-resolved computational fluid dynamics solutions, to predict the maximum lift coefficient for wing of arbitrary planform including the effects of twist and sweep. Fanjoy and Dorney [6] performed a series of numerical experiments, in which a Navier-Stokes analysis 
adapted for external flow was used to calculate the flow field around the dual-wing geometry and the stagger was varied, to study the tandem-airfoil interaction in different flight regimes. By solving the incompressible Navier-Stokes equation on overlapping grids, Broering and Lian [7] investigated the effect of wing spacing between fore and hind wings on the aerodynamics of a tandem flapping wing configuration numerically at Reynolds number of 5000. Zhang and Yu [8] investigated the unsteady aerodynamics of an aircraft with tandem wing configuration in the morphing stage using CFD methods. Most recently, Patidar et al. [9] investigated the lift and drag characteristics of a Busemann biplane with staggered tandem airfoil configurations at various Mach numbers between 0.5 and 2.5 using computational fluid dynamics. Computational fluid dynamics methods can satisfy the required accuracy with little financial cost but low computational efficiency.

Compared with tunnel experiment and the CFD method, theoretical calculation with little financial cost and high efficiency also has been widely used during the aircraft conceptual design stage. Prandtl's classical lifting-line theory [10] can be used to predict the section lift coefficient by obtaining an infinite series solution for the spanwise distribution of vorticity generated on the wing, which can compromise between the crude approximations provided by analytic methods as well as low-fidelity analyses and the high level of geometric detail, time, and resources required for the high-fidelity tools, such as Reynoldsaveraged Navier-Stokes computational fluid dynamics (CFD) methods, in the early aircraft conceptual design stage. With the assumption of the linear relationship between section lift and section angle of attack as well as the assumption of a straight lifting line, Prandtl's classical lifting-line theory can provide an analytical solution in the form of an infinite sine series for the circulation distribution to calculate the spanwise distribution of lift acting on a finite-lifting surface. For the evaluation of the coefficients in the sine series, Glauert [11] presented a very straightforward method. Moreover, a more rigorous and more rapidly converging method using Fourier series expansion was developed by Rasmussen and Smith [12]. Meanwhile, McCormick [13] proposed a purely numerical method to solve the liftingline equation for a single-lifting surface with a straight lifting line, and Anderson and Corda [14] improved the numerical method by relaxing the linear assumption of the relationship between section lift and section angle of attack. However, all the aforementioned methods, to obtain the solution to the classical lifting-line equation, only applied for the particular configuration with a single-lifting surface with no sweep and no dihedral.

For the presence of the flow interaction between the fore wing and the hind wing, the wake generated by the fore wing will be hampered by the hind wing, which will also change the effective angle of attack of the hind wing, so the aerodynamic characteristics of the tandem airfoil configuration are different from the single-wing case. For the tandem wing configuration or the configuration with multiple-lifting surface, Phillips and Snyder [15] presented a numerical lifting-line method, which was based on the Prandtl's original model of a finite wing, to predict the forces and moments acting on a system of lifting surfaces. In this numerical lifting-line method, the continuous distribution of section circulation was equivalent to a finite number of discrete horseshoe vortices approximately, which was referred to what proposed by Katz and Plotkin [16]. Furthermore, Spall et al. [17] calculated the solutions obtained from a numerical method based on Prandtl's lifting-line theory, valid for multiple-lifting surface with arbitrary sweep, for a number of rigid wing and geometries. Most recently, Jacobs et al. [18] extended the numerical lifting-line method to transonic speeds and application to multiple-lifting-surface configurations, such as wing-canard configuration and three-lifting-surface business jet. Thus, the accuracy of the methods used to obtain the solution to the lifting-line equation of tandem wing configuration or multiple-liftingsurface configuration depends on the finite number of discrete horseshoe vortices, and the section lift coefficient is not continuous along the wingspan.

In this paper, the main objective is to predict the section lift coefficient for the tandem wing configuration or multiplelifting-surface system. A lifting-line method based on Prandtl's classical lifting-line theory is proposed, in which the solution for the spanwise distribution of section circulation is expressed in the form of Fourier series truncated to a finite series. This approach can be used to obtain the lift acting on each lifting surface of tandem wing design and configuration with two lifting surfaces in arbitrary positions. Additionally, a series of tandem wing configuration with different parameters, including the gap, the stagger, the incidence angle, and the wingspan, are calculated and simulated using the proposed method to analyze the effect of the parameters on characteristics including lift and induced drag of each lifting surface. Results with this method are validated by experimental data and CFD results at small angle of attack. The accuracy from the presented method is shown to be comparable to that obtained from experiment and CFD with little experiment cost and computational cost.

\section{Lifting-Line Theory for Tandem Wing Configuration}

In this section, Prandtl's classical lifting-line theory for a single finite wing is presented firstly, and then, the liftingline theory for tandem wing configuration is proposed on the foundation of Prandtl's classical lifting-line theory combined with the Biot-Savart law.

2.1. Prandtl's Classical Lifting-Line Theory. Traditionally, the distribution of section circulation along the span for a single finite wing, with no sweep or dihedral and having an arbitrary spanwise variation in chord length, can be obtained by solving Prandtl's classical lifting-line equation. Prandtl's lifting-line theory provides a simple and precise spanwise distribution of section circulation for the special case of an elliptic wing. Otherwise, to solve Prandtl's lifting-line equation for the wing with arbitrary shape, the change of variable is very necessary. 


$$
y=-\frac{1}{2} b \cos (\theta)
$$

The variation in section circulation along the span of a finite wing, $\Gamma(\theta)$, as approximated by the infinite Fourier sine series solution to Prandtl's lifting-line equation, can be written as

$$
\Gamma(\theta)=2 b V_{\infty} \sum_{n=1}^{\infty} A_{n} \sin (n \theta) .
$$

Based on the Kutta-Joukowski theorem and the definition of the lift coefficient, the following relationship between the section circulation, $\Gamma(\theta)$, and the lift coefficient, $C_{\mathrm{L}}(\theta)$, is

$$
C_{\mathrm{L}}(\theta)=\frac{2 \Gamma(\theta)}{V_{\infty} c(\theta)} .
$$

Moreover, according to the assumption that the lift can be closely approximated as linear function of angle of attack for airfoil at small angle of attack, the section lift coefficient, $C_{\mathrm{L}}(\theta)$, can be approximately calculated as

$$
C_{\mathrm{L}}(\theta)=C_{\mathrm{L}, \alpha}\left(\alpha_{\mathrm{e}}(\theta)-\alpha_{0 \mathrm{~L}}\right)
$$

where $C_{\mathrm{L}, \alpha}$ is the airfoil section lift slope, $\alpha_{0 \mathrm{~L}}$ is the zero-lift angle of attack, and $\alpha_{\mathrm{e}}(\theta)$ is the effective local angle of attack.

Consequently, combining (3) and (4), the Prandtl's lifting-line equation applying on each lifting surface can be derived as

$$
\frac{2 \Gamma(\theta)}{V_{\infty} c(\theta) C_{\mathrm{L}, \alpha}}-\alpha_{\mathrm{e}}(\theta)=-\alpha_{0 \mathrm{~L}} .
$$

Prandtl's classical lifting-line equation can be solved by calculating the effective local angle of attack, $\alpha_{\mathrm{e}}(\theta)$, which can be obtained from the induced velocity generated by its self-downwash effect at a series of control points along the wingspan.

2.2. Application on Tandem Wing Configuration. For the tandem wing configuration, the variation in section circulation along the span of the fore wing and the hind wing, $\Gamma_{1}\left(\theta_{1}\right)$ and $\Gamma_{2}\left(\theta_{2}\right)$, as approximated by the infinite Fourier sine series solution to Prandtl's lifting-line equation, can be, respectively, written as

$$
\begin{aligned}
& \Gamma_{1}\left(\theta_{1}\right)=2 b_{1} V_{\infty} \sum_{n=1}^{\infty} A_{n} \sin \left(n \theta_{1}\right), \\
& \Gamma_{2}\left(\theta_{2}\right)=2 b_{2} V_{\infty} \sum_{n=1}^{\infty} B_{n} \sin \left(n \theta_{2}\right) .
\end{aligned}
$$

In order to distinguish the parameters, we defined some subscripts for the variables appeared in the flowing part, which can be explained in Table 1 .

According to the classical Prandtl's lifting-line theory, the bound vortex is located along the quarter chord, and the trailing vortex is parallel to the $x$-axis along the freestream velocity, as shown in Figure 1.
TABLE 1: Definition of the subscripts of the parameters.

\begin{tabular}{lc}
\hline Subscripts & Definition \\
\hline 1 & Parameters of the fore wing \\
11 & Parameters of the hind wing \\
12 & Parameters of the fore wing generated by itself \\
& Parameters of the fore wing generated \\
by the hind wing \\
21 & Parameters of the hind wing \\
22 & generated by the fore wing \\
$i$ & Parameters of the hind wing generated by itself \\
$x$ & The $x$-component of the parameters \\
$y$ & The $y$-component of the parameters \\
$z$ & The $z$-component of the parameters \\
-1 & Parameters generated by the bound vortex \\
-2 & Parameters generated by the trailing vortex \\
\hline
\end{tabular}

Considering the impact on aerodynamic between the two lifting surfaces and its self-downwash effect, the effective local angle of attack, $\alpha_{\mathrm{e} 1}\left(\theta_{1}\right)$ and $\alpha_{\mathrm{e} 2}\left(\theta_{2}\right)$, can be carried out as

$$
\begin{aligned}
& \alpha_{\mathrm{e} 1}\left(\theta_{1}\right)=\alpha+\delta_{1}-\varepsilon_{11}\left(\theta_{1}\right)-\varepsilon_{12}\left(\theta_{1}\right), \\
& \alpha_{\mathrm{e} 2}\left(\theta_{2}\right)=\alpha+\delta_{2}-\varepsilon_{22}\left(\theta_{2}\right)-\varepsilon_{21}\left(\theta_{2}\right),
\end{aligned}
$$

where $\alpha$ is the geometric angle of attack in an arbitrary spanwise section, $\delta_{1}$ and $\delta_{2}$ are the incidence angle of the fore wing and the hind wing, respectively, $\varepsilon_{11}\left(\theta_{1}\right)$ and $\varepsilon_{22}\left(\theta_{2}\right)$ are the induced angle of attack by its self-downwash effect, and $\varepsilon_{12}\left(\theta_{1}\right)$ and $\varepsilon_{21}\left(\theta_{2}\right)$ are the induced angle of attack by the upwash and downwash effect between the fore wing and the hind wing, respectively; the induced angle of attack is positive for the downwash effect.

Consequently, substituting (6) and (7) into (5), the Prandtl's lifting-line equation applying on each lifting surface can be derived as

$$
\begin{aligned}
& \frac{2 \Gamma_{1}\left(\theta_{1}\right)}{V_{\infty} c_{1}\left(\theta_{1}\right) C_{\mathrm{L} 1, \alpha}}+\varepsilon_{11}\left(\theta_{1}\right)+\varepsilon_{12}\left(\theta_{1}\right)=\alpha+\delta_{1}-\alpha_{0 \mathrm{~L}_{1}}, \\
& \frac{2 \Gamma_{2}\left(\theta_{2}\right)}{V_{\infty} c_{2}\left(\theta_{2}\right) C_{\mathrm{L} 2, \alpha}}+\varepsilon_{22}\left(\theta_{2}\right)+\varepsilon_{21}\left(\theta_{2}\right)=\alpha+\delta_{2}-\alpha_{0 \mathrm{~L}_{2}} .
\end{aligned}
$$

With the traditional aerodynamic theory on a finite wing with no sweep, the induced velocity generated by its own bound vortex at an arbitrary location along the lifting line can be ignored. As a consequence, only the induced velocity generated by the trailing vortex should be taken into consideration to calculate the induced angle of attack by its self-downwash effect. From the Biot-Savart 


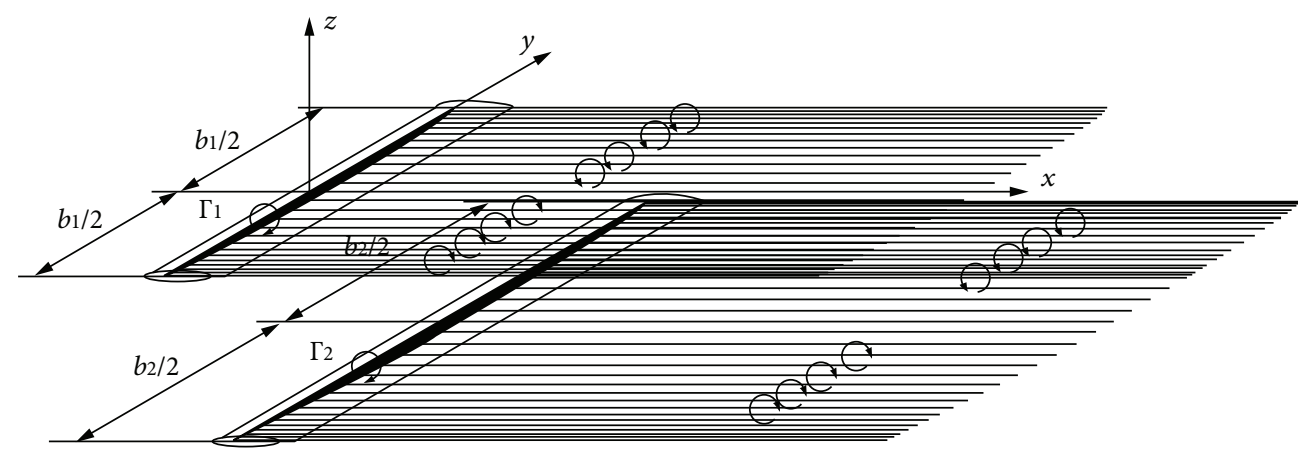

FIgURe 1: Prandtl's model for the bound vorticity and the trailing vortex parallel to the $x$-axis generated by tandem wings.

law, the $z$-velocity component induced by its own trailing vortex with the change of variable is

$$
\begin{aligned}
& V_{i z 11}\left(\theta_{1}\right)=V_{\infty} \sum_{n=1}^{\infty} \frac{n A_{n} \sin \left(n \theta_{1}\right)}{\sin \left(\theta_{1}\right)}, \\
& V_{i z 22}\left(\theta_{2}\right)=V_{\infty} \sum_{n=1}^{\infty} \frac{n B_{n} \sin \left(n \theta_{2}\right)}{\sin \left(\theta_{2}\right)} .
\end{aligned}
$$

Since the induced velocity is small compared to the freestream velocity, the induced angle of attack by its self-downwash effect, $\varepsilon_{11}\left(\theta_{1}\right)$ and $\varepsilon_{22}\left(\theta_{2}\right)$, would be approximated as the induced velocity divided by the freestream velocity, as

$$
\begin{aligned}
& \varepsilon_{11}\left(\theta_{1}\right)=\arctan \left(\frac{V_{i z 11}\left(\theta_{1}\right)}{V_{\infty}}\right) \approx \frac{V_{i z 11}\left(\theta_{1}\right)}{V_{\infty}}, \\
& \varepsilon_{22}\left(\theta_{2}\right)=\arctan \left(\frac{V_{i z 22}\left(\theta_{2}\right)}{V_{\infty}}\right) \approx \frac{V_{i z 22}\left(\theta_{2}\right)}{V_{\infty}} .
\end{aligned}
$$

In addition, the bound vortex as well as the trailing vortex generated by the other lifting surface can both produce an induced velocity at an arbitrary location along the lifting line. For the convenience of calculation, the $z$-velocity component induced by the horseshoe vortices generated by the other lifting surface would be separated into two parts, induced by the bound vortex and the trailing vortex. Applying the Biot-Savart law and the variable change, the $z$-velocity component induced by the bound vortex generated by the other lifting surface is

$$
\begin{aligned}
& V_{i z 12-1}\left(\theta_{1}\right)=-\cos (\varphi) \int_{0}^{\pi} \frac{\Gamma_{2}\left(\theta_{2}\right) \sin (\phi)}{4 \pi r^{2}}\left[\frac{b_{2}}{2} \sin \left(\theta_{2}\right)\right] d \theta_{2}, \\
& V_{i z 21-1}\left(\theta_{2}\right)=\cos (\varphi) \int_{0}^{\pi} \frac{\Gamma_{1}\left(\theta_{1}\right) \sin (\phi)}{4 \pi r^{2}}\left[\frac{b_{1}}{2} \sin \left(\theta_{1}\right)\right] d \theta_{1}
\end{aligned}
$$

where $\varphi$ is the angle between the induced velocity and the $z$-axis direction as shown in Figure $2, \cos (\varphi)=S_{\mathrm{t}}$ / $\left(S_{t}^{2}+G^{\prime 2}\right)^{1 / 2}, \varphi$ is the angle between the segment of the bound vortex and the line through the point and the vortex segment as shown in Figure $3, \sin (\phi)=\left(S_{t}^{2}+G^{\prime 2}\right)^{1 / 2} / r$,

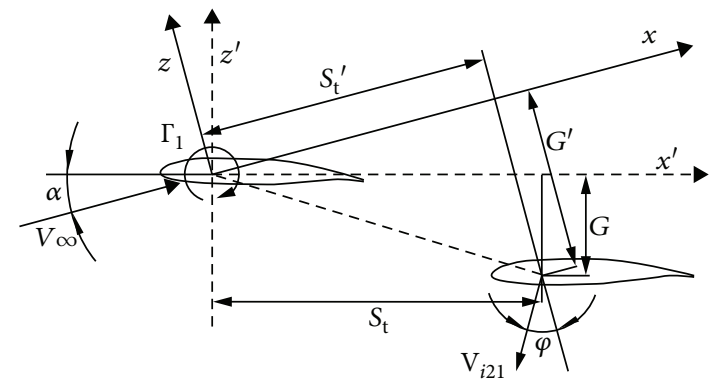

FIGURE 2: Relationship between the direction of the induced velocity and the $z$-axis.

$r$ is the distance from the point to the vortex segment, and $r=\left(\left(y_{2}-y_{1}\right)^{2}+S_{t}^{2}+G^{\prime 2}\right)^{1 / 2}, S_{t}$ is the longitudinal separation along the $x$-axis of the two wings and $G^{\prime}$ is the vertical distance along the $z$-axis between the two wings, as shown in Figure 2, and can be calculated with the coordinate transformation as

$$
\begin{aligned}
& S_{\mathrm{t}}^{\prime}=S_{\mathrm{t}} \cos (\alpha)+G \sin (\alpha), \\
& G^{\prime}=-S_{\mathrm{t}} \sin (\alpha)+G \cos (\alpha),
\end{aligned}
$$

where $S_{t}$ is the longitudinal separation of the two wings, stagger, and $G$ is the vertical distance between the two wings, gap, as shown in Figure 2.

Meanwhile, applying the Biot-Savart law into the segment of the trailing vortex and integral along the spanwise, the $z$-velocity component induced by the trailing vortex generated by the other lifting surface is

$$
\begin{aligned}
& V_{i z 12-2}\left(\theta_{1}\right)=\frac{1}{4 \pi} \int_{0}^{\pi} \frac{\delta y_{21}}{r^{\prime 2}}\left(1-\frac{S_{\mathrm{t}}}{r}\right) \frac{d \Gamma_{2}\left(\theta_{2}\right)}{d \theta_{2}} d \theta_{2}, \\
& V_{i z 21-2}\left(\theta_{2}\right)=\frac{1}{4 \pi} \int_{0}^{\pi} \frac{\delta y_{12}}{r^{\prime 2}}\left(1+\frac{S_{\mathrm{t}}}{r}\right) \frac{d \Gamma_{1}\left(\theta_{1}\right)}{d \theta_{1}} d \theta_{1},
\end{aligned}
$$

where $\delta y_{21}$ and $\delta y_{12}$ are the difference between the point and the trailing vortex segment in $y$ direction, $\delta y_{21}=y_{2}-y_{1}$ and $\delta y_{12}=y_{1}-y_{2}$, and $r^{\prime}$ is the perpendicular distance from the point to the trailing vortex filament, $r^{\prime}=\left(G^{\prime 2}+\delta y^{2}\right)^{1 / 2}$. 


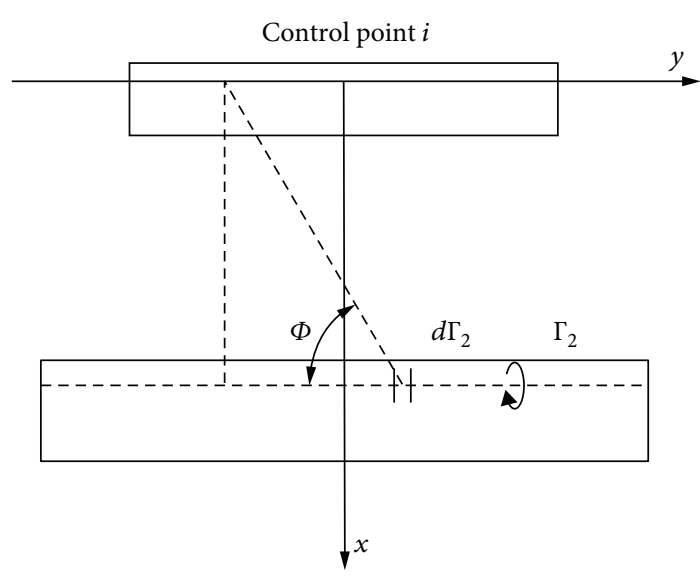

Figure 3: Definition of the angle describing the segment of the bound vortex and the vector.

Hence, with the same approximation in calculating the induced angle of attack by its self-downwash effect, the induced angle of attack by the other lifting surface would be

$$
\begin{aligned}
& \varepsilon_{12}\left(\theta_{1}\right)=\arctan \left(\frac{V_{i z 12-1}\left(\theta_{1}\right)+V_{i z 12-2}\left(\theta_{1}\right)}{V_{\infty}}\right) \approx \frac{V_{i z 12}\left(\theta_{1}\right)}{V_{\infty}}, \\
& \varepsilon_{21}\left(\theta_{2}\right)=\arctan \left(\frac{V_{i z 21-1}\left(\theta_{2}\right)+V_{i z 21-2}\left(\theta_{2}\right)}{V_{\infty}}\right) \approx \frac{V_{i z 21}\left(\theta_{2}\right)}{V_{\infty}} .
\end{aligned}
$$

Applying (9) to (14) into (8) at $N_{1}$ and $N_{2}$ points along the lifting line of the two lifting surfaces, respectively, the sequence $\left\{A_{n}\right\}$ and $\left\{B_{n}\right\}$ would be obtained by solving a system of $N$ (where $N=N_{1}+N_{2}$ ) linear equations and then the variation of section circulation along the span of the two lifting surfaces would be carried out.

Because of the wake-induced velocity, the freestream velocity vector will be rotated by the induced angle of attack, $\alpha_{i}$, as shown in Figure 4 and the induced angles of attack for the two wings can be obtained as

$$
\begin{aligned}
& \alpha_{i 1}\left(\theta_{1}\right)=\varepsilon_{11}\left(\theta_{1}\right)+\varepsilon_{12}\left(\theta_{1}\right), \\
& \alpha_{i 2}\left(\theta_{2}\right)=\varepsilon_{22}\left(\theta_{2}\right)+\varepsilon_{21}\left(\theta_{2}\right) .
\end{aligned}
$$

As we assumed that the induced velocity is small compared with the freestream velocity, then $\cos \alpha_{i}=1$ and $\sin \alpha_{i}=\alpha_{i}$, therefore the section lift coefficient would be calculated by using (3) easily, then the induced drag of the two wings, $D_{i 1}$ and $D_{i 2}$, can be written as

$$
\begin{aligned}
& D_{i 1}=\rho V_{\infty} \int_{0}^{\pi} \Gamma_{1}\left(\theta_{1}\right) \alpha_{i 1}\left(\theta_{1}\right)\left[\frac{b_{1}}{2} \sin \left(\theta_{1}\right)\right] d \theta_{1}, \\
& D_{i 2}=\rho V_{\infty} \int_{0}^{\pi} \Gamma_{2}\left(\theta_{2}\right) \alpha_{i 2}\left(\theta_{2}\right)\left[\frac{b_{2}}{2} \sin \left(\theta_{2}\right)\right] d \theta_{2} .
\end{aligned}
$$

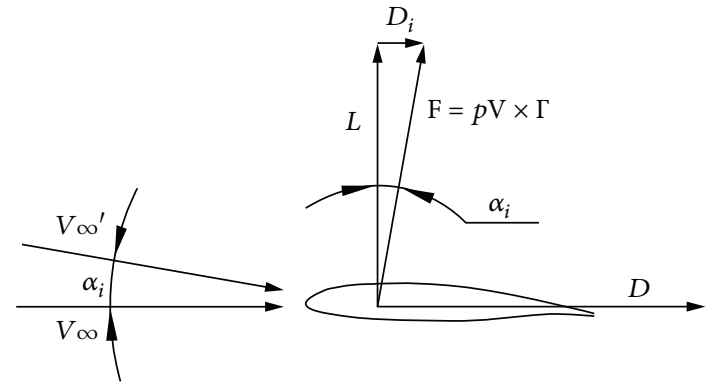

FIGURE 4: Schematic of the generation of the induced drag.

\section{Correction of the Magnitude of Local Velocity}

In the proceeding of calculating the section lift coefficients and the induced drag, the magnitudes of local velocity are both used in the Kutta-Joukowski theorem and the definition of the lift coefficient. To obtain the results closer to the real value, the magnitude of local velocity is corrected by that synthesized from the uniform freestream flow combined with the induced velocity at the corresponding control point.

3.1. Velocity Correction. Applying the Kutta-Joukowski theorem to a straight lifting surface, the force acting on a spanwise differential segment of the lifting surface related with the section circulation can be derived as

$$
d \mathbf{F}(\theta)=\rho \Gamma(\theta) \mathbf{V}(\theta) \times d \mathbf{l}
$$

According to previous statements, the velocity used in calculating the lift coefficient in (3) has been set to equal to the freestream velocity. However, because the circulation distributing along the lifting line as well as the relevant trailing vortex of each lifting surface can also induce velocity at the control points, the velocity used in (17) should be synthesized from the uniform freestream flow combined with the induced velocity at the control points and can be shown as

$$
\mathbf{V}(\theta)=V_{\infty}+\mathbf{V}_{i}(\theta)
$$

where $\mathbf{V}(\theta)$ is the vector of the local velocity at the control points and $\mathbf{V}_{i}(\theta)$ is the vector of the induced velocity at the control points generated by the other lifting surface, $\mathbf{V}_{i}(\theta)=\left(V_{i x}(\theta), V_{i y}(\theta), V_{i z}(\theta)\right)$.

Because the trailing vortex sheet parallel to the $x$-axis, so the $x$-velocity component induced by the trailing vortex equals to zero, the lift paralleled to the $z$-axis can be computed from (17) and (18) and the section lift coefficient of one lifting surface can be derived as

$$
C_{\mathrm{L}}(\theta)=\frac{|d \mathbf{F}(\theta)|}{1 / 2\left(\rho V_{\infty}^{2} c(\theta)|d \mathbf{l}|\right)}=\frac{2 V_{x}(\theta) \Gamma(\theta)}{V_{\infty}^{2} c(\theta)},
$$


where $V_{x}(\theta)$ is the local $x$-velocity component of one lifting surface composed of the freestream velocity and the $x$-velocity component induced by the bound vorticity of the other lifting surface, $V_{x 1}\left(\theta_{1}\right)=V_{\infty}+V_{i x 12-1}\left(\theta_{1}\right)$, $V_{x 2}\left(\theta_{2}\right)=V_{\infty}+V_{i x 21-1}\left(\theta_{2}\right)$.

Meanwhile, by applying (16) and the related equations, the induced drag coefficient of the two wings, $C_{D i 1}$ and $C_{D i 2}$, can be derived as

$$
\begin{aligned}
& C_{D_{i 1}}=\frac{2}{V_{\infty}^{2} S} \int_{0}^{\pi} \Gamma_{1}\left(\theta_{1}\right) \alpha_{i 1}\left(\theta_{1}\right) V_{x}\left(\theta_{1}\right)\left[\frac{b_{1}}{2} \sin \left(\theta_{1}\right)\right] d \theta_{1}, \\
& C_{D_{i 2}}=\frac{2}{V_{\infty}^{2} S} \int_{0}^{\pi} \Gamma_{2}\left(\theta_{2}\right) \alpha_{i 2}\left(\theta_{2}\right) V_{x}\left(\theta_{2}\right)\left[\frac{b_{2}}{2} \sin \left(\theta_{2}\right)\right] d \theta_{2},
\end{aligned}
$$

where $S$ is the wing area.

Applying the Biot-Savart law into the segment of the bound vortex along the lifting line of the other lifting surface and integral along the spanwise, the $x$-velocity component induced by the bound vortex at an arbitrary point along the lifting line is

$$
\begin{aligned}
& V_{i \times 12-1}\left(\theta_{1}\right)=-\sin (\varphi) \int_{0}^{\pi} \frac{\Gamma_{2}\left(\theta_{2}\right) \sin (\phi)}{4 \pi r^{2}}\left[\frac{b_{2}}{2} \sin \left(\theta_{2}\right)\right] d \theta_{2}, \\
& V_{i \times 21-1}\left(\theta_{2}\right)=\sin (\varphi) \int_{0}^{\pi} \frac{\Gamma_{1}\left(\theta_{1}\right) \sin (\phi)}{4 \pi r^{2}}\left[\frac{b_{1}}{2} \sin \left(\theta_{1}\right)\right] d \theta_{1},
\end{aligned}
$$

where $\sin (\varphi)=G^{\prime} /\left(S_{t}^{\prime 2}+G^{\prime 2}\right)^{1 / 2}$, as shown in Figure 2.

Moreover, the magnitude of the local velocity used in (10) and (14) to calculate the induced angle of attack should also be corrected by the local $x$-velocity component of each lifting surface composed of the freestream velocity and the induced $x$-velocity component, as shown in (21).
3.2. Solving Prandtl's Lifting-Line Equation. After replacing the magnitude of the freestream velocity with the corrected magnitude of the local velocity in the achievement of the section lift coefficient and the induced angle of attack, the classical Prandtl's lifting-line equation would be derived as a system of $N$ (where $N=N_{1}+$ $N_{2}$ ) nonlinear equation relating the $N$ unknown coefficients (consists of $\left\{A_{n}\right\}$ and $\left\{B_{n}\right\}$ ) of the distribution of the section circulation to known properties of the lifting surface.

Traditionally, the Newton's method is commonly adopted to solve the system of nonlinear equation. However, because the unknown coefficients exist both in the numerator and the denominator in the equations, the partial derivatives cannot be obtained conveniently. So, the Prandtl's lifting-line equation shown as (8) can be changed as

$$
\begin{aligned}
& \frac{2 V_{x 1}^{2}\left(\theta_{1}\right) \Gamma_{1}\left(\theta_{1}\right)}{V_{\infty}^{2} c_{1}\left(\theta_{1}\right) C_{\mathrm{L} 1, \alpha}}+V_{i z 11}\left(\theta_{1}\right)+V_{i z 12}\left(\theta_{1}\right) \\
& \quad=V_{x 1}\left(\theta_{1}\right)\left(\alpha+\delta_{1}-\alpha_{0 \mathrm{~L}_{1}}\right), \\
& \frac{2 V_{x 2}^{2}\left(\theta_{2}\right) \Gamma_{2}\left(\theta_{2}\right)}{V_{\infty}^{2} c_{2}\left(\theta_{2}\right) C_{\mathrm{L} 2, \alpha}}+V_{i z 22}\left(\theta_{2}\right)+V_{i z 21}\left(\theta_{2}\right) \\
& \quad=V_{x 2}\left(\theta_{2}\right)\left(\alpha+\delta_{2}-\alpha_{0 \mathrm{~L}_{2}}\right) .
\end{aligned}
$$

To apply the Newton's method, writing the first equation of (22) at $N_{1}$ different points and writing the second equation of (22) at $N_{2}$ different points, respectively, combining the unknown sequence, $\left\{A_{n}\right\},\left\{B_{n}\right\}$, as $\mathbf{X}=\left[A_{n}{ }^{T}, B_{n}{ }^{T}\right]$, then the system of $N$ nonlinear equation can be written in the vector form as

$$
T(\mathbf{X})=\mathbf{R},
$$

where

$$
T_{i}(\mathbf{X})= \begin{cases}\frac{2 V_{x 1}^{2}\left(\theta_{1 i}\right) \Gamma_{1}\left(\theta_{1 i}\right)}{V_{\infty}^{2} c_{1}\left(\theta_{1 i}\right) C_{\mathrm{L} 1, \alpha}}+V_{i z 11}\left(\theta_{1 i}\right)+V_{i z 12}\left(\theta_{1 i}\right)-V_{x 1}\left(\theta_{1 i}\right)\left(\alpha+\delta_{1}-\alpha_{0 L_{1}}\right), & 1 \leq i \leq N_{1}, \\ \frac{2 V_{x 2}^{2}\left(\theta_{2\left(i-N_{1}\right)}\right) \Gamma_{2}\left(\theta_{2\left(i-N_{1}\right)}\right)}{V_{\infty}^{2} c_{2}\left(\theta_{2\left(i-N_{1}\right)}\right) C_{\mathrm{L} 2, \alpha}}+V_{i z 22}\left(\theta_{2\left(i-N_{1}\right)}\right)+V_{i z 21}\left(\theta_{2\left(i-N_{1}\right)}\right)-V_{x 2}\left(\theta_{2\left(i-N_{1}\right)}\right)\left(\alpha+\delta_{2}-\alpha_{0 L_{2}}\right), & N_{1}+1 \leq i \leq N .\end{cases}
$$

In order to find the vector $\mathbf{X}$ of the $N$ unknown coefficients which makes all components of the residual vector $\mathbf{R}$ equal to zero, an initial estimate for the vector $\mathbf{X}$ is selected and iteratively refined by applying the Newton's corrector equation:

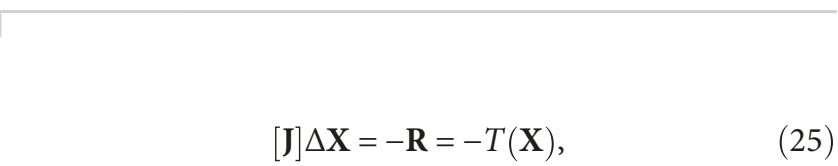

where $[\mathbf{J}]$ is the $N \times N$ matrix of partial derivatives. 


$$
J_{i j}=\frac{\partial T_{i}}{\partial X_{j}}= \begin{cases}\frac{2 V_{x 1}^{2}\left(\theta_{1 i}\right)}{V_{\infty}^{2} c_{1}\left(\theta_{1 i}\right) C_{\mathrm{L} 1, \alpha}} \frac{\partial \Gamma_{1}\left(\theta_{1 i}\right)}{\partial A_{j}}+\frac{\partial V_{i z 11}\left(\theta_{1 i}\right)}{\partial A_{j}}, & 1 \leq i \leq N_{1}, 1 \leq j \leq N_{1}, \\ \frac{4 \Gamma_{1}\left(\theta_{1 i}\right) V_{x 1}\left(\theta_{1 i}\right)}{V_{\infty}^{2} c_{1}\left(\theta_{1 i}\right) C_{\mathrm{L} 1, \alpha}} \frac{\partial V_{x 1}\left(\theta_{1 i}\right)}{\partial B_{\left(j-N_{1}\right)}}+\frac{\partial V_{i z 12}\left(\theta_{1 i}\right)}{\partial B_{\left(j-N_{1}\right)}}-\frac{\partial V_{x 1}\left(\theta_{1 i}\right)}{\partial B_{\left(j-N_{1}\right)}}\left(\alpha+\delta_{1}-\alpha_{0 \mathrm{~L}_{1}}\right), & 1 \leq i \leq N_{1}, N_{1}<j \leq N, \\ \frac{4 \Gamma_{2}\left(\theta_{2\left(i-N_{1}\right)}\right) V_{x 2}\left(\theta_{2\left(i-N_{1}\right)}\right)}{V_{\infty}^{2} c_{2}\left(\theta_{2\left(j-N_{1}\right)}\right) C_{\mathrm{L} 2, \alpha}} \frac{\partial V_{x 2}\left(\theta_{2\left(i-N_{1}\right)}\right)}{\partial A_{j}}+\frac{\partial V_{i z 21}\left(\theta_{2\left(i-N_{1}\right)}\right)}{\partial A_{j}}-\frac{\partial V_{x 2}\left(\theta_{2\left(i-N_{1}\right)}\right)}{\partial A_{j}}\left(\alpha+\delta_{2}-\alpha_{0 \mathrm{~L}_{2}}\right), & N_{1}<i \leq N, 1 \leq j \leq N_{1}, \\ \frac{2 V_{x 2}^{2}\left(\theta_{2\left(i-N_{1}\right)}\right)}{\partial \Gamma_{2}\left(\theta_{2\left(i-N_{1}\right)}\right)} \frac{\partial V_{i z 22}\left(\theta_{2\left(i-N_{1}\right)}\right)}{\partial B_{\left(j-N_{1}\right)}}, & N_{1}<i \leq N, N_{1}<j \leq N . \\ V_{\infty}^{2} c_{2}\left(\theta_{2\left(i-N_{1}\right)}\right) C_{\mathrm{L} 2, \alpha} & \end{cases}
$$

Furthermore, the correction vector can be calculated by applying (26) in (25), and the improved estimate of the vector $\mathbf{X}$ of the $N$ unknown coefficients in the $(k+1)$ th iteration based on the correction vector $\Delta \mathbf{X}$ and the vector $\mathbf{X}$ obtained in the $k$ th iteration can be carried out as

$$
\mathbf{X}^{(k+1)}=\mathbf{X}^{(k)}+\Omega \Delta \mathbf{X}^{(k)} .
$$

This iterating process repeated until satisfying the convergence condition as

$$
\frac{\left\|\Delta \mathbf{X}^{(k)}\right\|}{\left\|\mathbf{X}^{(k)}\right\|} \leq \xi,
$$

then the vector $\mathbf{X}$ of the $N$ unknown coefficients equals to the vector $\mathbf{X}^{(k)}$.

For the purpose of fast convergence of the Newton's method, an accurate initial estimate of the vector $\mathbf{X}$ of the $N$ unknown coefficients should be meticulously selected. So, it is appropriate to use the solution of the classical Prandtl's lifting-line equation regardless of the correction of the magnitude of local velocity as shown in (8).

After determining the circulation strength of each lifting surface, the aerodynamic force can be obtained by evaluating the integral of the aerodynamic force coefficient indicated as (19) along the wingspan of each lifting surface.

\section{Methods to Generate Control Points}

In order to calculate the Fourier coefficients for tandem wing configuration, the Prandtl's lifting-line equation, (8), must be applied at the control points of each lifting surface along its wingspan and then, the variation of the section circulation for the two lifting surface along the wingspan can be obtained.

Firstly, the simplest method to generate control points is that the lifting surface can be divided into elements of equal spanwise increment. In one manner, it would seem most reasonable to place the control point of each element along its bound vortex segment, midway between the two edges of each element, as shown in Figure 5.
Furthermore, for the pressure difference near the wing tip, the vorticity is shed from the wing more rapidly in the region near the wing tip, the control points should be clustered more tightly in this region to get best calculation efficiency. From [15], the conventional cosine clustering method could be used to generate element for control points of the two wings in tandem wing configuration. For the straight wing, the clustering method is only needed in the region near the wing tip. While for the wing with sweep and/or dihedral, the clustering method is needed both in the region near the wing tip and near the wing root since a step change in the slop of the quarter chord exists at the root for that wing, which caused the downwash to change very rapidly in the region near the wing root.

For the convenience that the method to generate control point can be appropriate for both the wing with/without sweep and/or dihedral, the clustering method should be applied independently over each semispan of each wing, as shown in Figure 6. Based on the variable change indicated in (1), the variables, $\theta_{1}$ and $\theta_{2}$, vary from 0 to $\pi$ as $y_{1}$ varies from $-b_{1} / 2$ to $b_{1} / 2$, as well as $y_{2}$ varies from $-b_{2} / 2$ to $b_{2} / 2$ over each wing. Distributing the dividing line for the junctures of adjacent element uniformly in variable $\theta$ will provide the expected clustering in variable $y$.

After gathering the clustering elements over each wing, there are two different methods to get the location of the control point of each element. Firstly, it would seem most appropriately to set the control point at the midway between the two nodal of each element, on the bound segment of the vortex. Then, the position of the control point $i$ can be easily obtained by applying the coordinates of the node $i-1$ and the node $i$. Secondly, another method has been used by Phillips and Snyder [15] to generate the control point based on the clustering. It may be more properly to achieve the control points by setting the coordinate midway in variable $\theta$ instead of midway in variable $y$.

Considering the interaction between the flow generated by the two lifting surfaces, control points in the region directly before or behind the wing tip region of the other lifting surface should also be clustered for the more accurate 


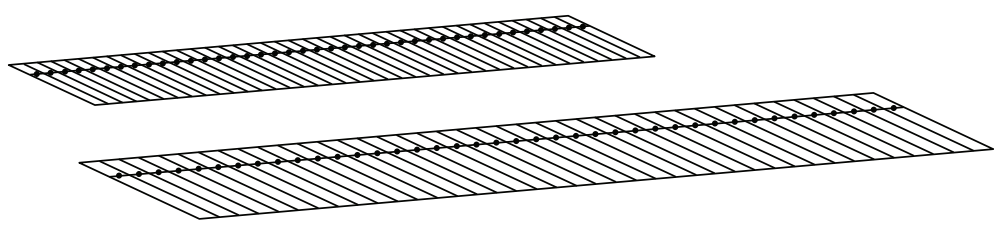

FIGURE 5: Grid generation for equal spanwise increment.

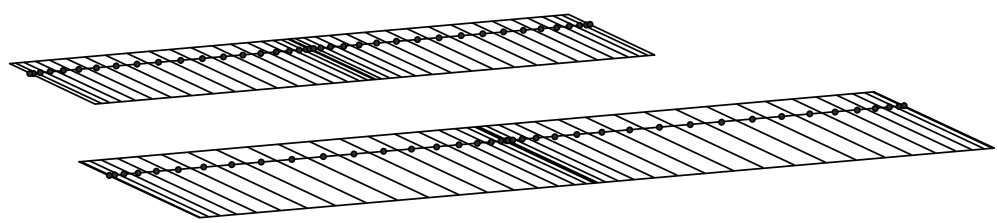

FIGURE 6: Grid generation with cosine clustering.

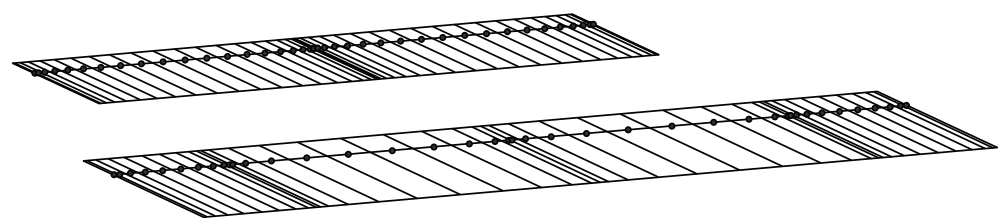

FIGURE 7: Grid generation with independent clustering.

section lift coefficients. For the tandem wing configuration with different wingspans of the two lifting surface, the cosine clustering method should be applied independently both in the region near the wing tip and wing root as well as the region aforementioned, as shown in Figure 7.

After validating and comparing, the clustering method with the midway in variable $\theta$ is selected to calculate the section lift coefficient for tandem wing configuration or multiple-lifting-surface system.

\section{Results}

5.1. Validation of the Prandtl's Lifting-Line Method. To validate the method for predicting the section lift coefficient of the tandem wing configuration or configuration with two lifting surfaces using the Prandtl's lifting-line theory and to analyze the grid sensitivity of the number of the control points, the section lift coefficient of tandem wing configuration by solving Prandtl's lifting-line equation at a series of different numbers of control points has been calculated and compared with the results obtained from an inviscid CFD solution and a limited experimental data.

For the experimental data comparison, the wing-canard configuration test cases presented by Feistel et al. [3] are selected. The experiment was conducted in the NASA Ames $7 \times 10$ foot wind tunnel in which the Reynolds number based on chord was $1.4 \times 10^{6}$ and the dynamic pressure was $60 \mathrm{lb} / \mathrm{ft}^{2}$, in which the magnitude of the freestream velocity was about $69.15 \mathrm{~m} / \mathrm{s}$. The tested models were constructed from the NASA/LANGLEY LS(1)-0413 (GA(W)-2) airfoil section, and the canard had an incidence of $2^{\circ}$ relative to the wing. The experiment data of the short-stagger, high-

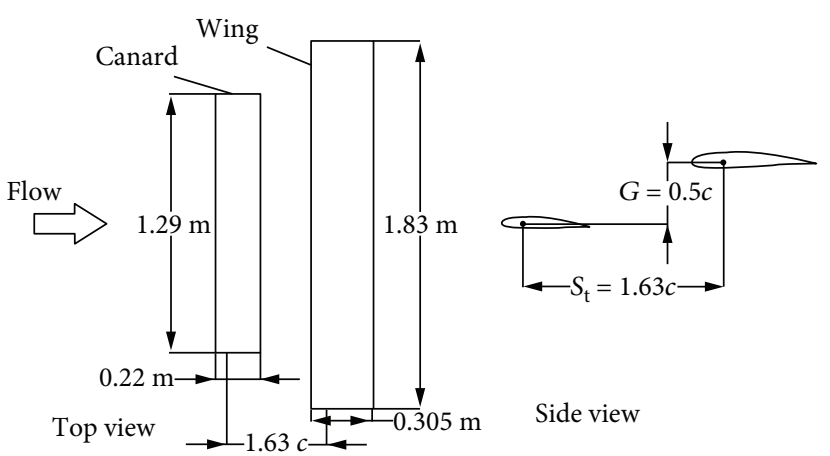

FIgURE 8: Wing-canard model top view and side view.

wing setup configuration was chosen for the significant interference effects between the wing and the canard presented in the wind tunnel.

The CFD solutions are performed by applying a structured-grid, multizone code, which solves the threedimensional, time-independent, Navier-Stokes equations using the finite volume method and SST (shear stress transport) $k-\omega$ turbulence model [19], for a rectangular tandem wing configuration, as shown in Figure 8. All aerodynamic simulations are calculated using $\mathrm{O}$-grid generation about tandem wing configuration, which is clustered in the normal direction near the wing surface to capture the boundary layer conveniently. Velocity-in and pressure-out boundary conditions are defined on the far-field planes of the computational flow field. The flow condition used in CFD solutions is the same as that in the wind tunnel experimental conducted by Feistel et al. in [3]. 


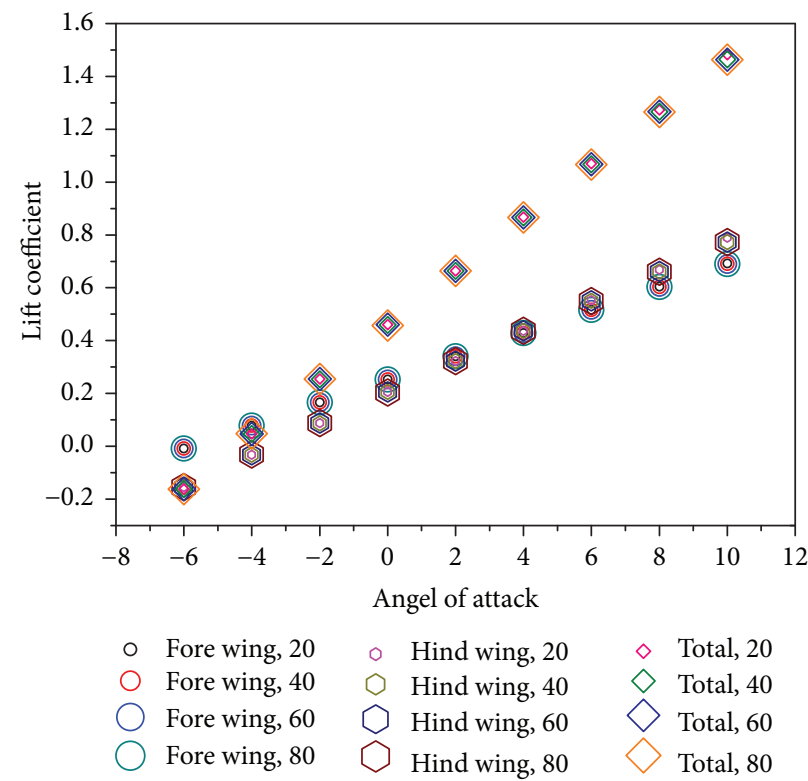

(a) Total lift coefficient

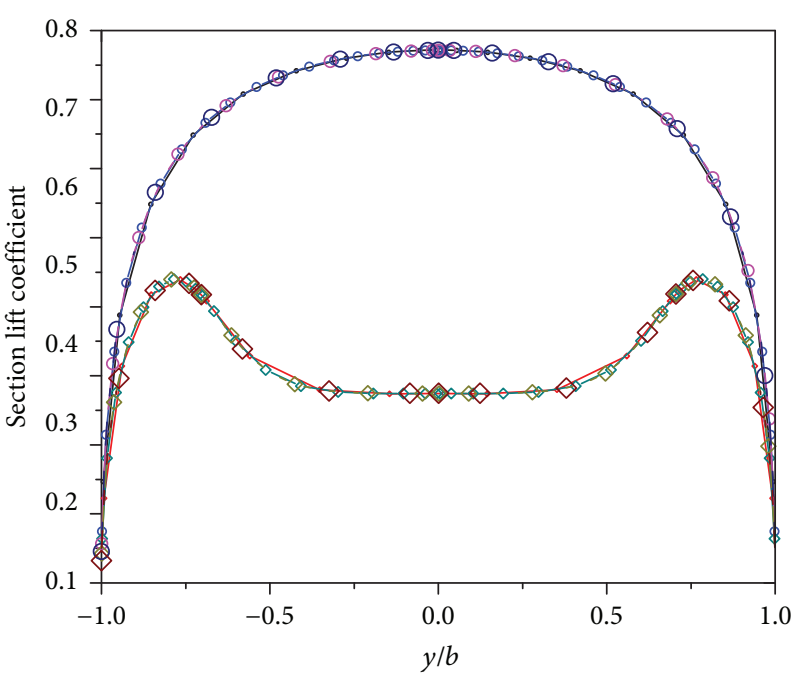

$$
\begin{array}{ll}
-\bullet-\text { Fore wing, } 20 & -\bullet-\text { Hind wing, } 20 \\
\text { - - F Fre wing, } 40 & -\diamond-\text { Hind wing, } 40 \\
\text {-०- Fore wing, 60 } & -\diamond-\text { Hind wing, 60 } \\
\text { ○- Fore wing, } 80 & -\checkmark \text { Hind wing, } 80
\end{array}
$$

(b) Section lift coefficient

FIgURE 9: Grid sensitivity test.

In addition, the number of control points along the semispan of each lifting surface is set from 20 to 80 with an increment of 20 to analyze the grid sensitivity. The model used to calculate is constructed from the NASA/LANGLEY LS(1)-0413 (GA(W)-2) airfoil section and with the configuration as shown in Figure 8 . Results of the section lift coefficient and the total lift coefficient of each lifting surface in tandem wing configuration are indicated in Figure 9.

As shown in Figure 9, the influence on the section lift coefficient and the wing lift coefficient by the number of control points per semispan can be ignored, then we set 40 control points per semispan for the best compromise between speed and accuracy.

The wing lift coefficient of the tandem wing configuration shown in Figure 8 is analyzed by comparing with the CFD solutions and the experiment data which were obtained with the same flow condition. Additional validations for the tandem wing configurations constructed from Wortmann FX63-137 airfoil by Scharpf and Mueller [4], and JEDEJ75 airfoil by Zhang and Yu [8], are conducted using the CFD method as well as the method presented in this paper with the same flow conditions in [4] and [8], respectively, and the results are indicated in Figure 10. As shown in Figure 11, the lift coefficient of the individual lifting surface is not predicted accurately by the lifting-line method compared with CFD and experiment data, while the total lift coefficient agrees well with the wind tunnel data and the CFD results over the majority of angles of attack in the range investigated. Furthermore, the lifting-line method proposed in this paper can predict the lift coefficient of the individual lifting surface as well as the total lift coefficient of the tandem wing configurations in $[4,8]$. The deviation of the lift coefficients obtained by the lifting-line method and CFD method is acceptable, which is not more than $5 \%$ in the majority of angles of attack investigated, which indicated in Figure 10.

To verify whether the method on the foundation of Prandtl's classical lifting-line theory can estimate the lift coefficient of a whole configuration including the airframe, the three-dimensional model with the airframe is simulated by using the CFD method. As shown in Figure 12, the length of the model airframe, $L$, is $1.25 \mathrm{~m}$, and the dimension of the cross airframe is $0.129 \times 0.129 \mathrm{~m}$. The NACA4309 airfoil is used as the fore wing and the hind wing. The main geometric characteristics of the two wings are a constant chord, $c$, of $0.129 \mathrm{~m}$ across the span, a reference wing area, $S$, of $0.544767 \mathrm{~m}^{2}$, the fore wing's span, $b_{1}$, of $2.1805 \mathrm{~m}$, and the hind wing's span, $b_{2}$, of $2.0425 \mathrm{~m}$. The UAV model is simplified from a folding missile-carried vehicle, and the stagger, $S_{t}$, must be large enough to fit the entire wing span. Hence, $S_{\mathrm{t}}$ is chosen as $0.89 \mathrm{~m}$ and $G$ is $-0.101 \mathrm{~m}$. The lift coefficient are evaluated at freestream $V=30 \mathrm{~m} / \mathrm{s}$, corresponding to a Reynolds number, Re, of $2.65 e^{5}$ based on the mean aerodynamic chord. Velocity-in and pressure-out boundary conditions are defined on the far-field planes of the computational flow field.

As shown in Figure 13, when the angle of attack is below 6 degrees, the numerical results obtained by the lifting-line method are in excellent agreement with the CFD data for the tandem wing configuration with/without airframe, which indicates that the airframe has little inference on the lift coefficient. However, the method based on Prandtl's liftingline theory cannot estimate the lift coefficient of the whole configuration with airframe accurately anymore when the angle of attack is above 6 degrees. Nevertheless, the liftingline method can also be extensively applied during the 


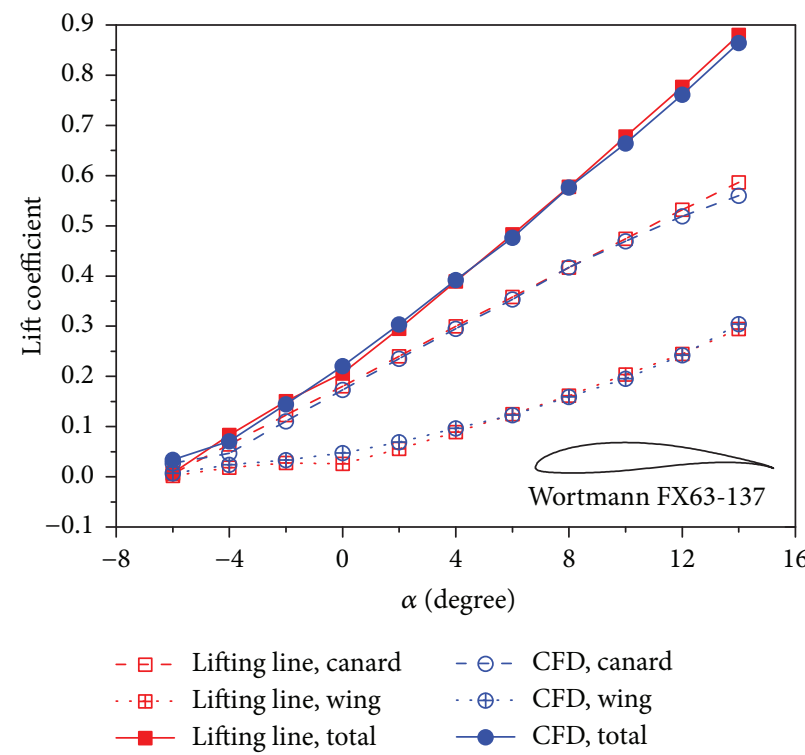

(a) Configuration with Wortmann FX63-137 airfoil

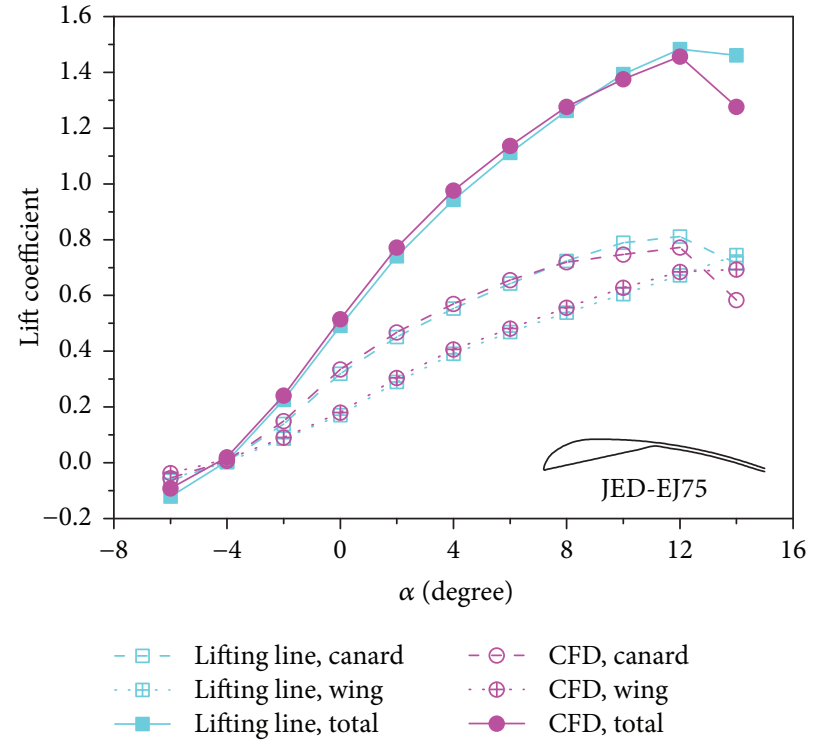

(b) Configuration with JED-EJ75 airfoil

FIGURE 10: Validation for different tandem wing configurations.

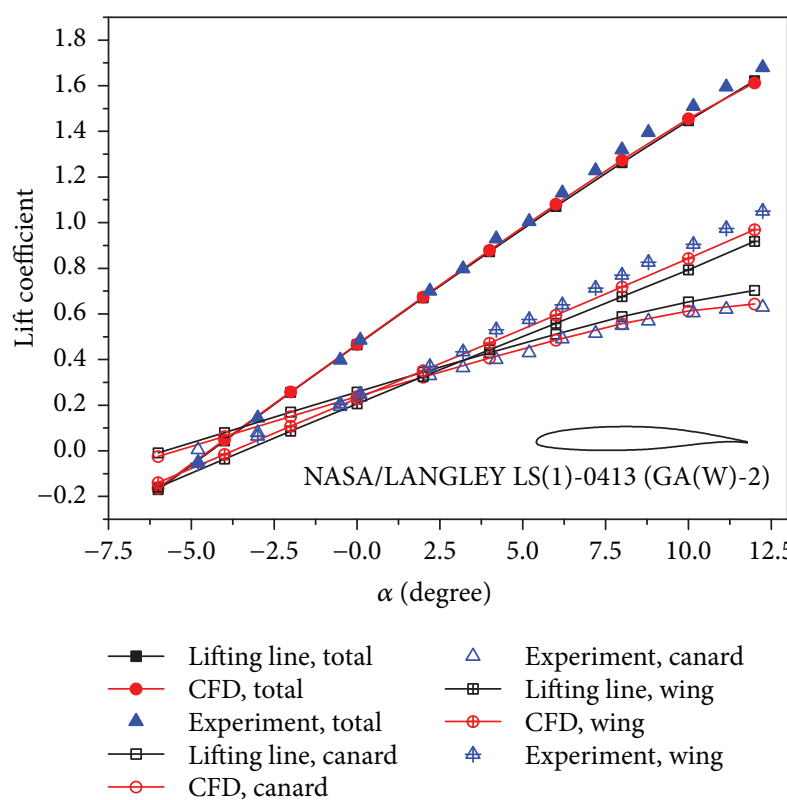

FIGURE 11: Lift curve comparison for tandem wing configuration.

aircraft conceptual design stage to obtain the aerodynamic characteristics of an aircraft with tandem wing configuration more efficiently.

5.2. Result Analysis. For tandem wing configuration, the gap represents the vertical distance between the aerodynamic centers of the two different lifting surfaces and the stagger represents the horizontal distance between the two different lifting surfaces' aerodynamic center. As the lifting-line method indicated, the induced velocity at the control points generated by the bound vortex and the trailing vortex of the other lifting surface would vary with the gap and the stagger. Meanwhile, the different incidence angle as well as the wingspan of one lifting surface would influence the region and the direction of its trailing vortex sheet, respectively, and further influence the induced velocity.

The section lift coefficient, the total lift coefficient, and the coefficient of the induced drag of each lifting surface of tandem wing configuration with different gap, stagger, incidence angle, and wingspan are calculated and analyzed by using the method proposed. All the simulations are calculated at freestream $V=69.15 \mathrm{~m} / \mathrm{s}$, corresponding to a Reynolds number of $1.4 \times 10^{6}$ based on chord, with the CFD method in the validation. The NASA/LANGLEY LS(1)-0413 (GA(W)-2) airfoil section is selected to construct all the tandem wing configurations, and the number of control points per semispan is set as 40 for the best compromise between speed and accuracy.

5.2.1. Effect of Gap. Effect of the gap can be analyzed from the section lift coefficient along the wingspan of each lifting surface for the tandem wing configuration, in which the stagger is $1.63 c, S_{\mathrm{t}}=1.63 c$, the incidence angle along its wingspan is constant, $\delta_{1}=2^{\circ}, \delta_{2}=0$, and the gap ranges from $-2.0 c$ to $2.0 c$. The results are calculated at angle of attack as 2 degrees, by using the Prandtl's classical method proposed above, and shown in Figure 14 and Table 2.

As shown in Figure 14, the section lift coefficient of the fore wing decreases along the entire wingspan with the vertical distance of the two lifting surfaces increasing, and it has a little bigger value with the positive gap, for the reason that the upwash effect by the hind wing becomes weaken. On the other hand, the section lift coefficient of the hind wing increases along the wingspan in the region directly behind the fore wing, and it is bigger with the negative gap, while it declines somewhat along the other wingspan region and has a smaller value with the negative gap, because the downwash effect by the fore wing weakens with the gap increasing. What is more, as Table 2 indicated, the wing lift coefficient 


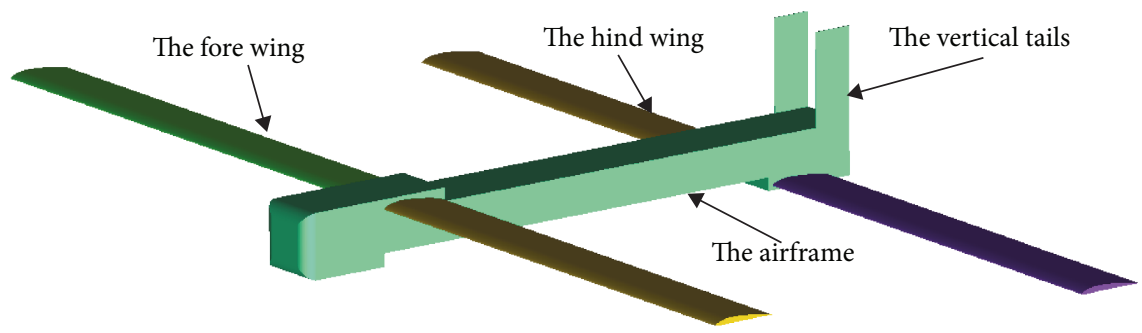

FIgURE 12: Model of tandem wing configuration with airframe.

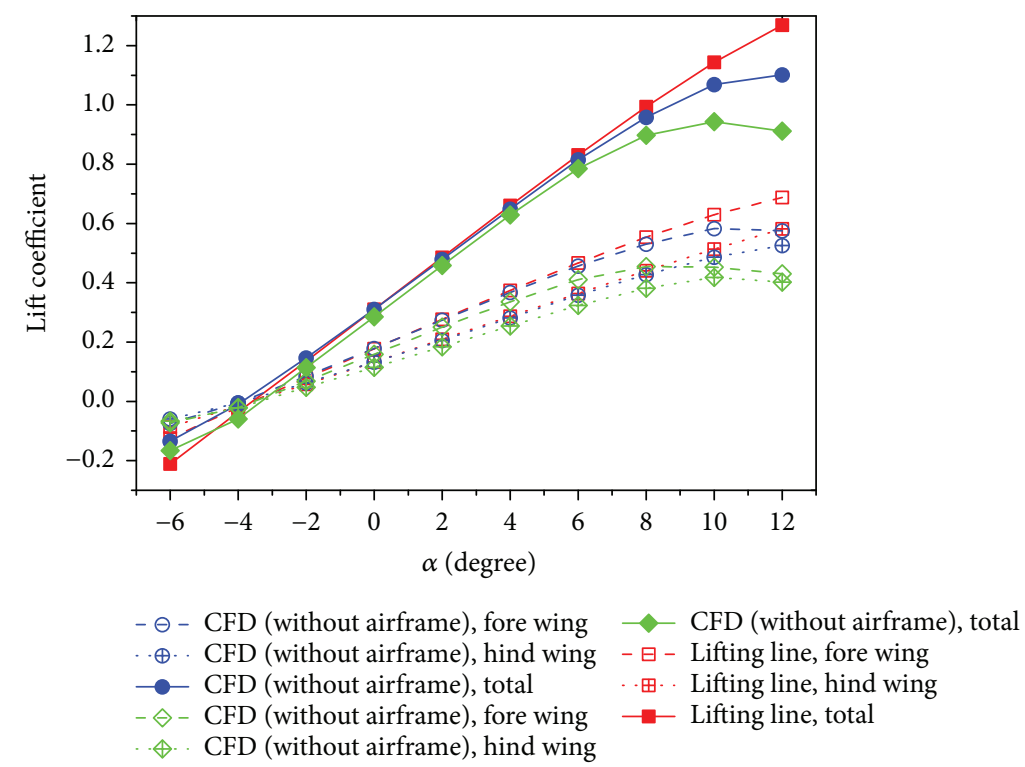

FIGURE 13: Lift curve comparison for tandem wing configuration with airframe.

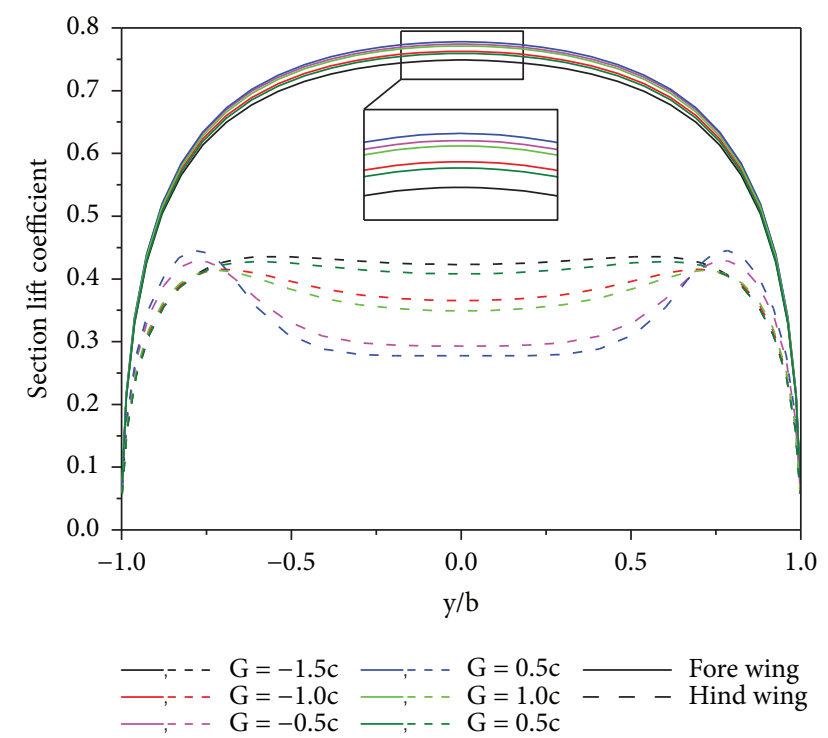

FIGURE 14: Effect of gap on section lift coefficient for tandem wing configuration.

has the biggest value for the fore wing with the small positive gap but for the hind wing as well as the tandem wing configuration with the big negative gap. Because the upwash effect generated by the hind wing can decrease the induced angle of attack of the fore wing, the induced drag of the fore wing is small when the two wings locate closer to each other in the vertical direction, as shown in Table 2 . However, the induced drag of the hind wing and the whole configuration increase firstly and decrease subsequently with the gap increasing both in positive and negative directions. That is because the downwash effect produced by the fore wing on the hind wing is in the dominant situation for the whole tandem wing configuration.

5.2.2. Effect of Stagger. The range of stagger from $1.0 c$ to $7.0 c$ is calculated to analyze its effect on the section lift coefficient, the wing lift coefficient and the coefficient of the induced drag. As shown in Figure 15 and Table 2, the section lift coefficient as well as the wing lift coefficient of the fore wing decrease with the stagger increasing, but it is opposite for its induced drag and for that of the hind wing, and it tends to be constant gradually, for the reason that the aerodynamic interaction between the two lifting surfaces becomes weaken. Meanwhile, the total lift coefficient of the tandem wing configuration becomes larger with the stagger increasing as shown in Table 2.

5.2.3. Effect of Incidence Angle. For the effect of incidence angle, a series of tandem wing configurations with the range of incidence angle from -6 to 6 degrees of one lifting surface 
TABLE 2: Lift coefficient and coefficient of induced drag with the effect of stagger and gap.

\begin{tabular}{lcccccccccccccc}
\hline$G$ & $C_{\mathrm{L} 1}$ & $C_{\mathrm{L} 2}$ & $C_{\mathrm{L}}$ & $C_{D i 1}$ & $C_{D i 2}$ & $C_{D i}$ & $S_{\mathrm{t}}$ & $C_{\mathrm{L} 1}$ & $C_{\mathrm{L} 2}$ & $C_{\mathrm{L}}$ & $C_{D i 1}$ & $C_{D i 2}$ & $C_{D i}$ \\
\hline$-1.5 c$ & 0.3325 & 0.4036 & 0.7361 & 0.0113 & 0.0168 & 0.0281 & $1.0 c$ & 0.3542 & 0.2944 & 0.6486 & 0.0099 & 0.0168 & 0.0267 \\
$-1.0 c$ & 0.3383 & 0.3723 & 0.7106 & 0.0108 & 0.0174 & 0.0283 & $2.0 c$ & 0.3408 & 0.3371 & 0.6779 & 0.0109 & 0.0173 & 0.0281 \\
$-0.5 c$ & 0.3433 & 0.3337 & 0.6769 & 0.0105 & 0.0174 & 0.0279 & $3.0 c$ & 0.3345 & 0.3522 & 0.6867 & 0.0113 & 0.0172 & 0.0286 \\
$0.5 c$ & 0.3447 & 0.3262 & 0.6709 & 0.0106 & 0.0172 & 0.0278 & $4.0 c$ & 0.3315 & 0.3583 & 0.6894 & 0.0115 & 0.0172 & 0.0287 \\
$1.0 c$ & 0.3415 & 0.3635 & 0.7050 & 0.0109 & 0.0175 & 0.0284 & $5.0 c$ & 0.3298 & 0.3612 & 0.6910 & 0.0117 & 0.0171 & 0.0288 \\
$1.5 c$ & 0.3364 & 0.3950 & 0.7315 & 0.0113 & 0.0169 & 0.0282 & $6.0 c$ & 0.3288 & 0.3629 & 0.6917 & 0.0117 & 0.0171 & 0.0288 \\
\hline
\end{tabular}

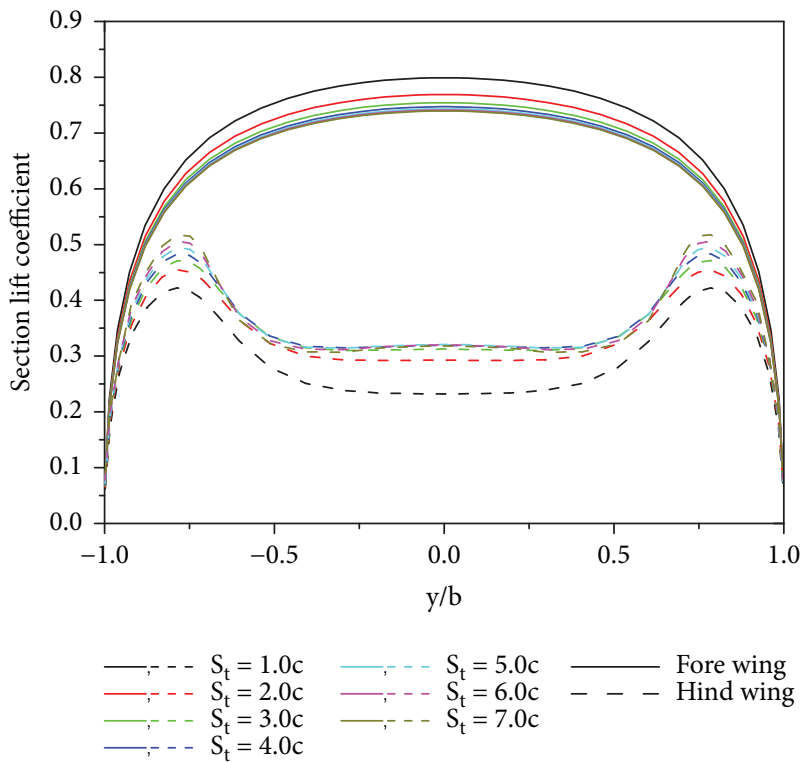

Figure 15: Effect of stagger on section lift coefficient for tandem wing configuration.

and the constant incidence angle of the other lifting surface are calculated, the section lift coefficients of the two lifting surfaces are shown in Figure 16, and the total lift coefficient and the coefficient of the induced drag of each lifting surface with the variation of the incidence angle of the other lifting surface are shown in Table 3.

The bigger incidence angle of the hind wing will produce the better section lift coefficient and smaller induced drag of the fore wing, for the reason that the upwash effect has been strengthened to increase the effective angle of attack of the fore wing section. However, the section lift coefficient of the hind wing decreases along the wingspan in the region directly behind the fore wing with the incidence angle of the fore wing increasing while it increases somewhat in the other wingspan region, because the direction of the wake stream is changed with the incidence angle of the fore wing increasing. The induced drag of the hind wing has the bigger value when the incidence angle of the fore wing is about zero, and it decreases with the magnitude of the incidence angle of the fore wing increasing.

5.2.4. Effect of Wingspan. When the wingspan of the fore wing, $b_{1}$, is bigger than the constant wingspan of the hind wing, $b_{2}$, the hind wing is in the region of the fore wing's wake stream entirely and is influenced along its whole wingspan. As shown in Figure 17(a), the section lift coefficient of the hind wing is positively correlated with the wingspan of the fore wing. If the wingspan of the fore wing, $b_{1}$, is smaller than the constant wingspan of the hind wing, $b_{2}$, the region influenced by the fore wing's wake is different with the change of the wingspan of the fore wing, so the section lift coefficient of the hind wing decreases with the wingspan of the fore wing increasing and the curve peak moves towards to the middle of the hind wing. As Table 3 shown, the lift coefficient of the hind wing is smallest when the wingspans of the two lifting surfaces are almost equal, but the induced drag of the hind wing is biggest when the two wings have the almost equal wingspan, and it continues to increase with the wingspan of the fore wing increasing, which is bigger than that of the hind wing.

As Figure 17(b) and Table 3 indicated, the wingspan of the hind wing has little influence on the section lift coefficient of the fore wing, and there is a positive correlation between the section lift coefficient of the fore wing and the wingspan of the hind wing, but it is opposite for that of the induced drag of the fore wing.

5.2.5. Effect of Wing Planform. Effects on the section lift coefficient and the induced drag by different parameters discussed above are all based on that the canard as well as the wing is straight rectangular wing. In this section, the effects of the wing platform, such as the taper ratio, AR, as well as the aspect ratio, $\lambda$, on the lift coefficient and the induced drag are analyzed with the wing-canard configuration indicated in Figure 8. A series of simulation cases, in which the taper ratio of one lifting surface varies from 0 to 1 based on its unchanged root chord with an unchanged taper ratio of another lifting surface, are calculated to analyze the effect of the taper ratio. What is more, the effect of the aspect ratio is discussed with the calculation cases, in which the aspect ratio of the one lifting surface varies while its wingspan remains constant and the aspect ratio of another lifting surface keeps unchanged.

As shown in Figure 18, the taper ratio of the fore wing has a significant effect on the lift coefficient of the hind wing, the lift coefficient of the hind wing decreases with the taper ratio of the fore wing increasing, but it is opposite for that of the fore wing, when the taper ratio is about 0.6 , and the effects on the lift coefficient are similar to that by the elliptical wing. With the taper ratio of the hind wing increasing, the upwash 


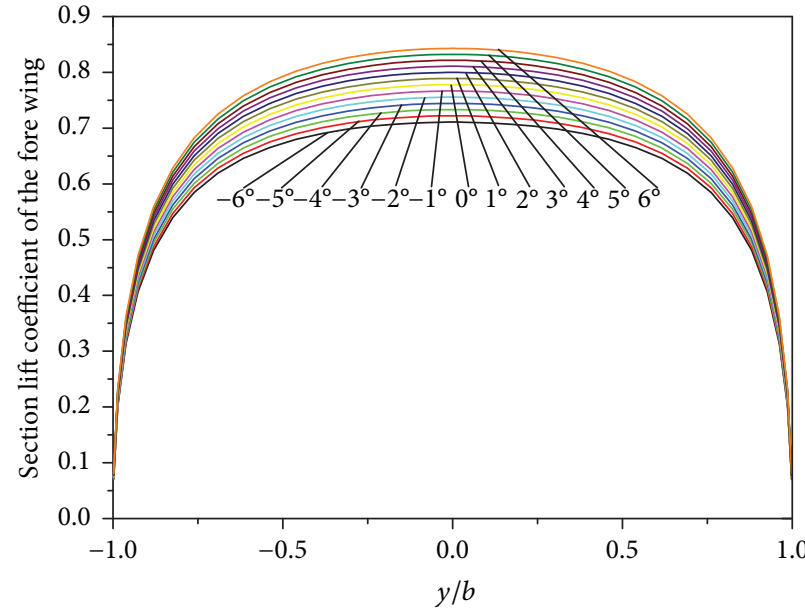

(a) Incidence angle of the hind wing

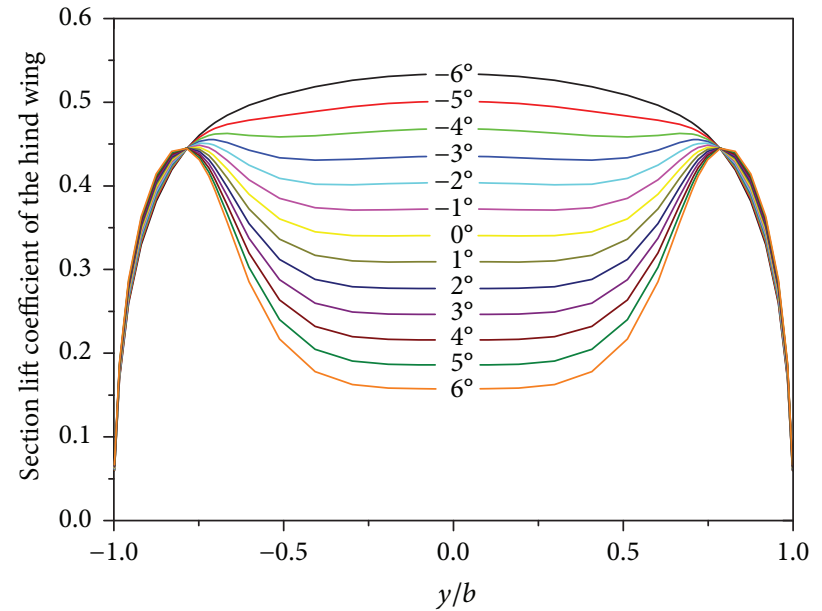

(b) Incidence angle of the fore wing

FIGURE 16: Effect of incidence angle on section lift coefficient for tandem wing configuration.

TABLE 3: Lift coefficient and coefficient of induced drag with the effect of incidence angle and wingspan.

\begin{tabular}{lcccccccccccc}
\hline$\delta_{1}$, degree & $C_{\mathrm{L} 2}$ & $C_{D i 2}$ & $\delta_{2}$, degree & $C_{\mathrm{L} 1}$ & $C_{D i 1}$ & $b_{2}, \mathrm{~m}$ & $C_{\mathrm{L} 2}$ & $C_{D i 2}$ & $b_{2}, \mathrm{~m}$ & $C_{\mathrm{L} 1}$ & $C_{D i 1}$ \\
\hline-6 & 0.4739 & 0.0140 & -6 & 0.3163 & 0.0125 & 0.99 & 0.3711 & 0.0166 & 0.53 & 0.6475 & 0.0795 \\
-5 & 0.4550 & 0.0150 & -5 & 0.3210 & 0.0122 & 1.09 & 0.3543 & 0.0168 & 0.63 & 0.6491 & 0.0665 \\
-4 & 0.4361 & 0.0158 & -4 & 0.3257 & 0.0119 & 1.19 & 0.3408 & 0.0170 & 0.73 & 0.6508 & 0.0571 \\
-3 & 0.4173 & 0.0164 & -3 & 0.3305 & 0.0116 & 1.29 & 0.3262 & 0.0172 & 0.83 & 0.6525 & 0.0499 \\
-2 & 0.3990 & 0.0169 & -2 & 0.3352 & 0.0113 & 1.39 & 0.3113 & 0.0173 & 0.93 & 0.6542 & 0.0443 \\
-1 & 0.3809 & 0.0173 & -1 & 0.3399 & 0.0109 & 1.49 & 0.2967 & 0.0174 & 1.03 & 0.6560 & 0.0398 \\
0 & 0.3626 & 0.0174 & 0 & 0.3447 & 0.0106 & 1.59 & 0.2836 & 0.0201 & 1.43 & 0.6658 & 0.0276 \\
1 & 0.3444 & 0.0174 & 1 & 0.3494 & 0.0102 & 2.09 & 0.2538 & 0.0155 & 1.53 & 0.6686 & 0.0257 \\
2 & 0.3260 & 0.0172 & 2 & 0.3540 & 0.0098 & 2.19 & 0.2580 & 0.0164 & 1.63 & 0.6718 & 0.0239 \\
3 & 0.3078 & 0.0169 & 3 & 0.3587 & 0.0094 & 2.29 & 0.2621 & 0.0165 & 1.73 & 0.6750 & 0.0223 \\
4 & 0.2899 & 0.0164 & 4 & 0.3633 & 0.0090 & 2.39 & 0.2663 & 0.0166 & 1.83 & 0.6780 & 0.0208 \\
5 & 0.2724 & 0.0157 & 5 & 0.3679 & 0.0086 & 2.49 & 0.2703 & 0.0167 & 1.93 & 0.6808 & 0.0195 \\
6 & 0.2553 & 0.0150 & 6 & 0.3724 & 0.0082 & 2.59 & 0.2741 & 0.0168 & 2.03 & 0.6835 & 0.0184 \\
\hline
\end{tabular}

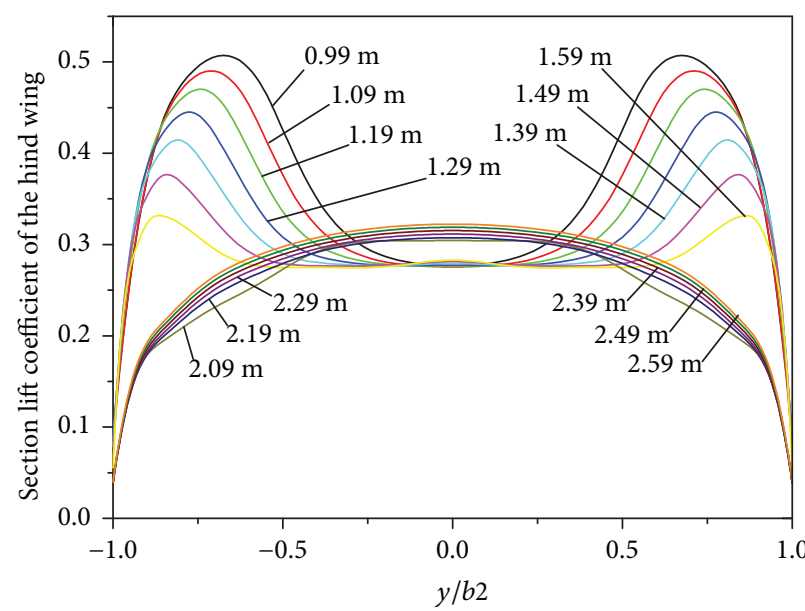

(a) Wingspan of the fore wing

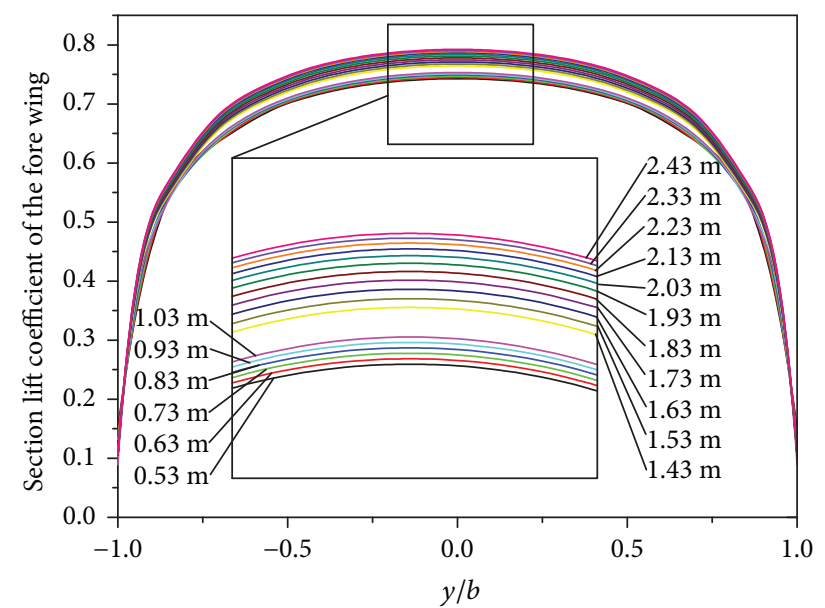

(b) Wingspan of the hind wing

FIGURE 17: Effect of wingspan on section lift coefficient for tandem wing configuration. 


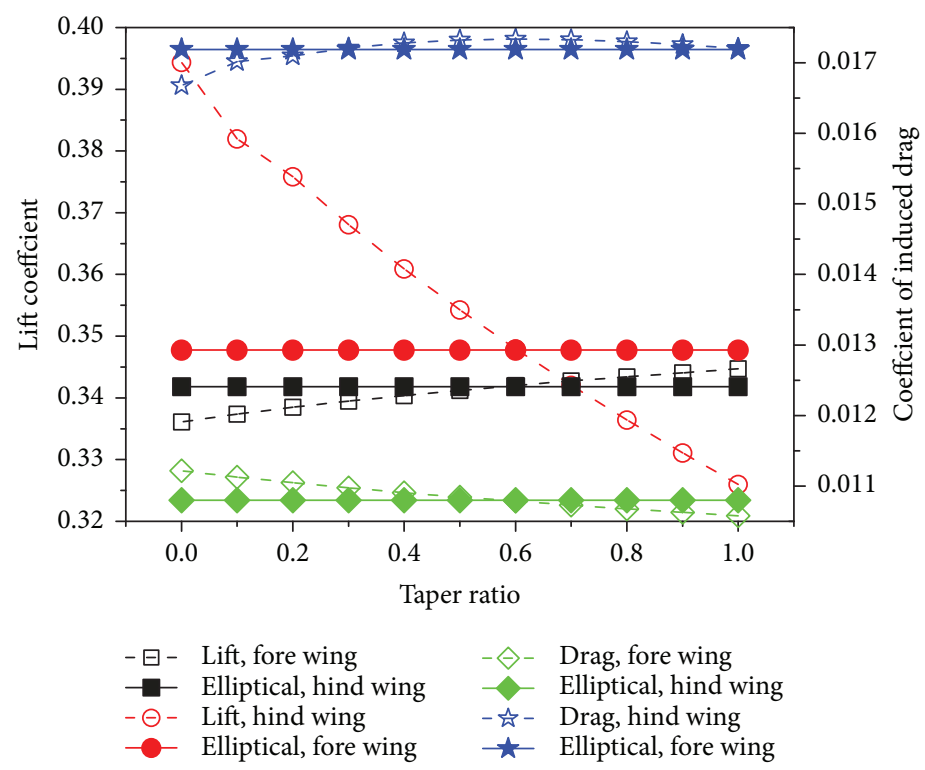

FIGURE 18: Effect of taper ratio on lift coefficient and induced drag for tandem wing configuration.

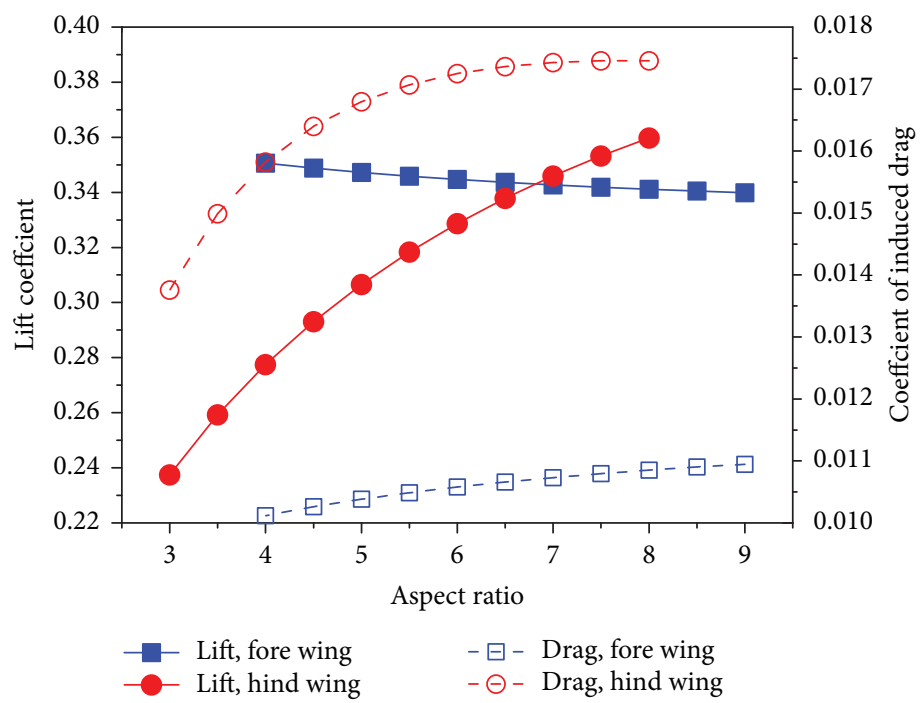

FIGURE 19: Effect of aspect ratio on lift coefficient and induced drag for tandem wing configuration.

effect becomes strengthened, so the induced drag of the fore wing decreases. The hind wing has the biggest induced drag when the taper ratio of the fore wing is about 0.6 , while it has the smallest induced drag when the planform of the fore wing is triangular.

In order to separate the effect of the wingspan in the calculation cases to analyze the effect of the aspect ratio, the wingspan of the lifting surface is set as unchanged and the aspect ratio varies, which is equivalent to the chord of the lifting surface changes. With the aspect ratio of the fore wing increasing, both the lift coefficient and the induced drag of the hind wing increase as shown in Figure 19. On the other hand, the variation of the aspect ratio of the hind wing has slightly influenced on the lift coefficient and induced drag of the fore wing. When the aspect ratio of the hind wing increases (i.e., the chord of the hind wing decreases), the lift coefficient of the fore wing decreases slightly, whereas the coefficient of the induced drag increases slightly.

Note from all results in Figures 10-19, the lift coefficient of the hind wing is much lower than that of the fore wing, which is because that an interference phenomenon appears in the flow field around the two wings. Nevertheless, the induced drag for tandem airfoil configuration will be smaller with an appropriate location of the two wings compared to the traditional layout. Additionally, the wingspan of the wings in tandem wing configuration can be reduced to obtain enough stiffness, with the same lift coefficient of the present configuration for the reason that the two wings in tandem airfoil configuration are all generating positive lift. Meanwhile, the position of the aerodynamic center of tandem wing configuration moves backward compared with that of the traditional layout, 
so that it is more competitive to carry payloads. In conclusion, tandem wing configuration can be widely used in missile-carried vehicle as well as gun-launching vehicles with folding wings especially.

\section{Conclusions}

(1) In accordance with Prandtl's classical lifting-line theory, a method to calculate the section lift coefficient for the tandem wing configuration or multiple-lifting-surface system is presented and the form of Fourier sine series is used to express the variation of the section circulation which changes continuously along the wingspan.

(2) To obtain the results closer to the real value, the magnitude of local velocity is corrected by that synthesized from the uniform freestream flow combined with the induced velocity at the corresponding control point.

(3) For configuration with different wingspans of the two lifting surfaces, the cosine clustering method is applied independently in the region near the wing tip and wing root as well as the region directly before or behind the wing tip region of the other lifting surface.

(4) The accuracy of the numerical solutions obtained by the proposed method is validated by the data obtained from computation fluid dynamics and tunnel experiment.

(5) From the results of tandem wing configuration with different parameters, it indicates that the bigger negative gap and stagger can produce better lift characteristic for tandem wing configuration or multiple-lifting-surface system. What is more, it will also be beneficial for the lift characteristic when the incidence angle and the wingspan of fore wing are appropriately declined or if the incidence angle and the wingspan of hind wing are appropriately increased.

(6) The induced drag of tandem wing configuration is dominated by the downwash effect generated by the fore wing on the hind wing. Furthermore, the minimum value of induced drag can be obtained by optimizing the parameters which can influence the aerodynamic interaction between the two wings.

\section{Conflicts of Interest}

The authors declared that there is no conflict of interest regarding the publication of this paper.

\section{References}

[1] J. W. Bottomley, "Tandem wing aircraft," Aerospace, vol. 4, pp. 12-20, 1977.
[2] J. W. Bottomley, "The tandem wing concept applied to modern transports," Aeronautical Journal, vol. 78, pp. 523524, 1974.

[3] T. Feistal, V. Corsiglia, and D. Levin, "Wind-tunnel measurements of wing-canard interference and a comparison with various theories," in SAE Technical Paper Series, pp. 1-14, Wichita, KS, USA, 1981, , Society of Automotive Engineers.

[4] D. F. Scharpf and T. J. Mueller, "Experimental study of a low Reynolds number tandem airfoil configuration," Journal of Aircraft, vol. 29, no. 2, pp. 231-236, 1992.

[5] N. R. Alley, W. F. Phillips, and R. E. Spall, "Predicting maximum lift coefficient for twisted wings using computational fluid dynamics," Journal of Aircraft, vol. 44, no. 3, pp. 911-917, 2007.

[6] D. Fanjoy and D. Dorney, "A study of tandem-airfoil interaction in different flight regimes," in 35th Aerospace Sciences Meeting and Exhibit, pp. 1-18, Reno, NV, USA, January 1997, AIAA Inc.

[7] T. Broering and Y. Lian, "The effect of wing spacing on tandem wing aerodynamics," in 28th AIAA Applied Aerodynamics Conference, pp. 1-20, Chicago, IL, USA, June 2010, AIAA Inc.

[8] G. Q. Zhang and S. C. M. Yu, "Unsteady aerodynamics of a morphing tandem-wing unmanned aerial vehicle," Journal of Aircraft, vol. 49, no. 5, pp. 1315-1323, 2012.

[9] V. K. Patidar, R. Yadav, and S. Joshi, "Numerical investigation of the effect of stagger on the aerodynamic characteristics of a Busemann biplane," Aerospace Science and Technology, vol. 55, pp. 252-263, 2016.

[10] L. Prandtl, "Applications of modern hydrodynamics to aeronautics," Report No.: NACA 116, Göttingen University, 1921.

[11] H. Glauert, The Elements of Aerofoil and Airscrew Theory, Cambridge University Press, Cambridge, UK, 2nd edition, 1983.

[12] M. L. Rasmussen and D. E. Smith, "Lifting-line theory for arbitrarily shaped wings," Journal of Aircraft, vol. 36, no. 2, pp. 340-348, 1999.

[13] B. W. McCormick, "The lifting line model," in Aerodynamics, Aeronautics and Flight Mechanics, pp. 340-348, Wiley, New York, NY, USA, 2nd edition, 1995.

[14] J. D. Anderson and S. Corda, "Numerical lifting-line theory applied to drooped leading-edge wings below and above stall," Journal of Aircraft, vol. 17, no. 12, pp. 898-904, 1980.

[15] W. F. Phillips and D. O. Snyder, "Modern adaptation of Prandtl's classic lifting-line theory," Journal of Aircraft, vol. 37, no. 4, pp. 662-670, 2000.

[16] J. Katz and A. Plotkin, "Lifting-line solution by horseshoe elements," in Low-Speed Aerodynamics from Wing Theory to Panel Methods, pp. 379-386, McGraw-Hill, New York, NY, USA, 1991.

[17] R. E. Spall, W. F. Phillips, and B. B. Pincock, "Numerical analysis of multiple, thin-sail geometries based on Prandtl's lifting-line theory," Computers \& Fluids, vol. 82, pp. 29-37, 2013.

[18] R. Jacobs, H. Ran, M. Kirby, and D. Mavris, "Extension of a modern lifting-line method to transonic speeds and application to multiple-lifting-surface configurations," in 30th AIAA Applied Aerodynamics Conference, pp. 787-799, New Orleans, LA, USA, June 2012, AIAA Inc.

[19] F. R. Menter, "Two-equation eddy-viscosity turbulence models for engineering applications," AIAA Journal, vol. 32, no. 8 , pp. $1598-1605,1994$. 


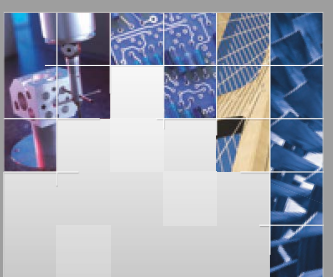

\section{Enfincering}
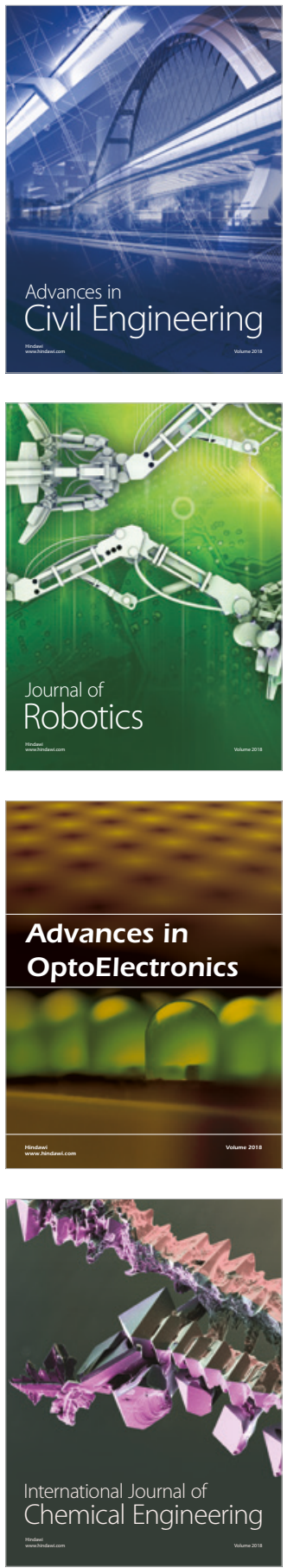

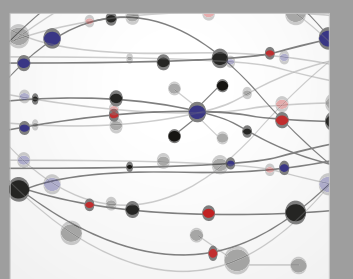

\section{Rotating \\ Machinery}

The Scientific World Journal

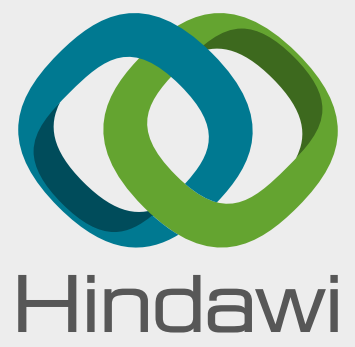

Submit your manuscripts at

www.hindawi.com
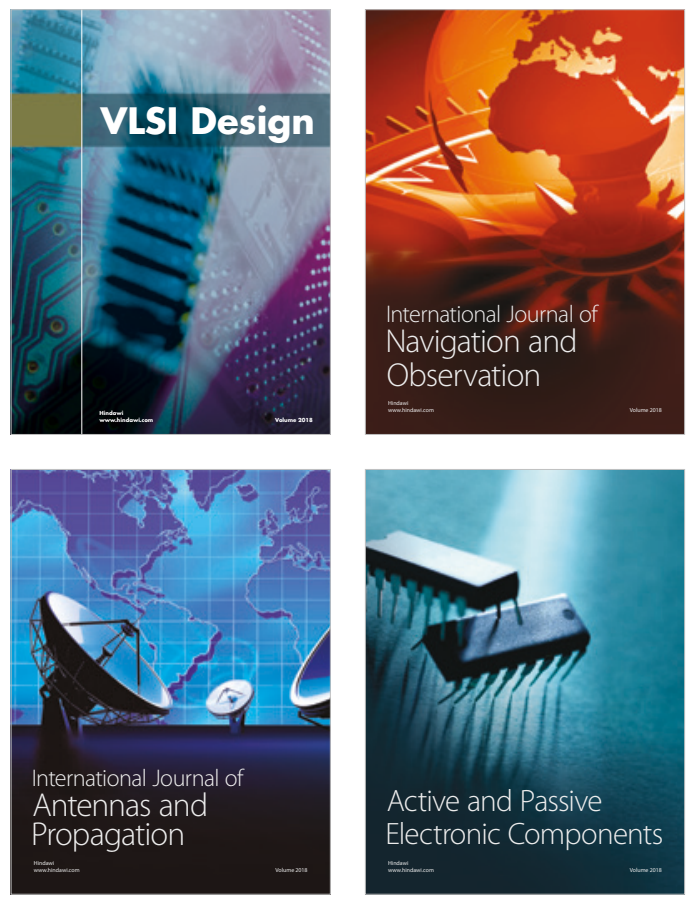
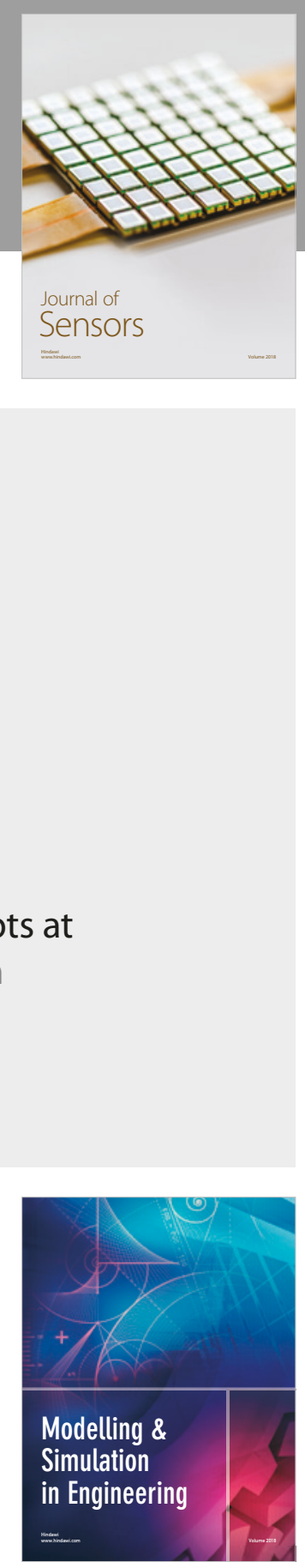

\section{Advances \\ Multimedia}
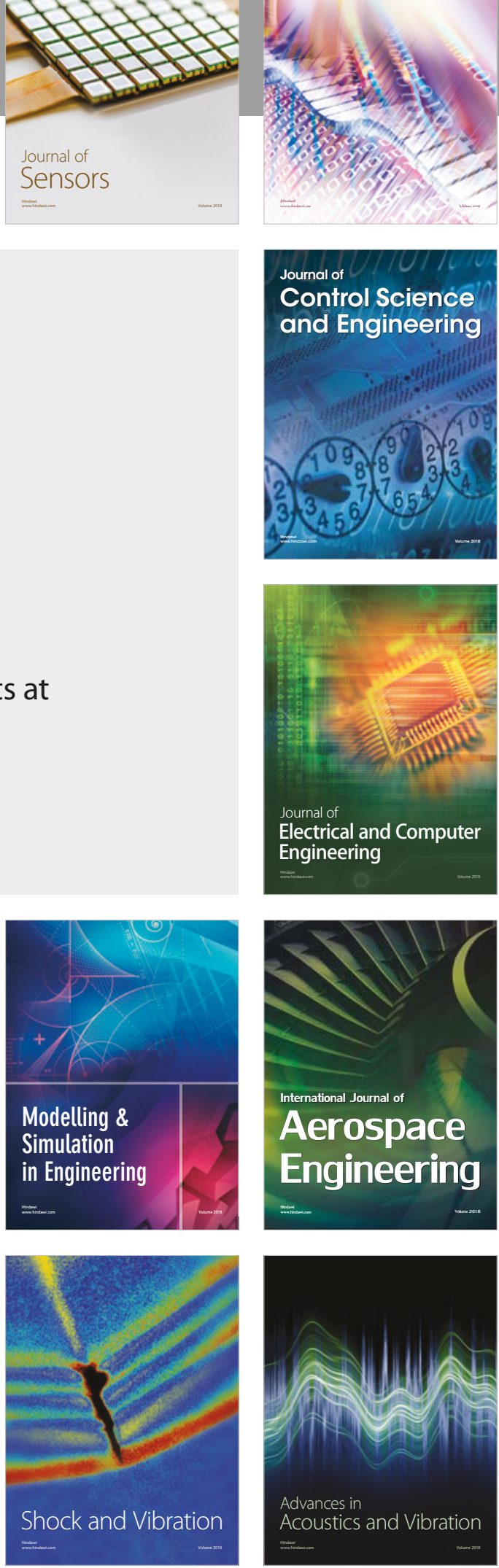